山्山FRANÇAISE

$>\mathrm{DE}$

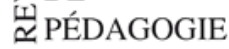

Revue française de pédagogie

Recherches en éducation

199 | avril-mai-juin 2017

L'histoire des disciplines : un champ de recherche en mutation

\title{
Des disciplines en recomposition? Heurs et malheurs d'une réforme du curriculum au collège (1988-1989)
}

Curriculum reform in lower-secondary education in France. Fortunes and misfortunes: 1988-1989

Pierre Clément

\section{(2) OpenEdition}

Journals

Édition électronique

URL : https://journals.openedition.org/rfp/6097

DOI : $10.4000 / r f p .6097$

ISSN : 2105-2913

Éditeur

ENS Éditions

Édition imprimée

Date de publication : 30 juin 2017

Pagination : 95-116

ISBN : 979-10-362-0108-0

ISSN : 0556-7807

Référence électronique

Pierre Clément, «Des disciplines en recomposition? Heurs et malheurs d'une réforme du curriculum au collège (1988-1989) », Revue française de pédagogie [En ligne], 199 | avril-mai-juin 2017, mis en ligne le 30 juin 2020, consulté le 06 janvier 2022. URL : http://journals.openedition.org/rfp/6097 ; DOI : https://doi.org/10.4000/rfp.6097 


\title{
Des disciplines en recomposition? Heurs et malheurs d'une réforme du curriculum au collège (1988-1989)
}

\author{
Pierre Clément
}

\begin{abstract}
Cet article étudie les enjeux de la recomposition des champs disciplinaires entre la nomination de Lionel Jospin au ministère de l'Éducation nationale en mai 1988 et le vote de la loi d'orientation du 14 juillet 1989. À partir de fonds d'archives variés et d'entretiens, il montre en quoi cette réforme du curriculum est encastrée dans des enjeux budgétaires, statutaires, syndicaux et politiques. Cet encastrement se joue notamment autour du projet de créer un corps spécifique de professeurs de collège dont la compétence pédagogique ne correspondrait ni aux bivalences des professeurs d'enseignement général de collège (PEGC), ni aux spécialités disciplinaires des certifiés. Après avoir rappelé les origines syndicales de ce projet et expliqué ce qui a poussé Lionel Jospin à y renoncer, cet article montre de quelle façon la recomposition des champs disciplinaires s'est alors imposée comme le principal levier de la réforme de cette école moyenne de masse qu'est le collège.
\end{abstract}

Mots-clés (TESE) : tronc commun, réforme des programmes d'études, contenu de l'éducation, approche interdisciplinaire, syndicat, statut de l'enseignant

\begin{abstract}
Lors de la séquence politique qui s'ouvre avec la victoire du Parti socialiste (PS) aux élections présidentielles et législatives de 1988, l'inadaptation des contenus d'enseignement s'impose à nouveau comme un problème public, c'est-à-dire comme " "quelque chose" à propos de quoi "quelqu'un" doit faire quelque chose » (Gusfield, 2009, p.5). Alors que le bilan du précédent gouvernement socialiste a été marqué par la cristallisation de l'idée de crise de l'école (Balland, 2009), par l'échec sur la question laïque, avec l'abandon du projet «Service public unifié et laïque de l'Éducation natio-
\end{abstract}

nale», et par les premières conséquences de la deuxième explosion scolaire (Poullaouec \& Lemêtre, 2009), la transformation des programmes apparaît comme I'un des principaux axes d'une politique scolaire qui se voudrait à la fois progressiste et modernisatrice (Clément, 2012).

Le nouveau ministre de l'Éducation nationale, Lionel Jospin, et son conseiller spécial, Claude Allègre, prennent en effet une série d'initiatives qui ambitionnent de transformer en profondeur les contenus d'enseignement : création de commissions dites 
«verticales» ou "thématiques», chargées de réfléchir aux évolutions des programmes discipline par discipline de la maternelle à l'enseignement supérieur; mise en place d'une commission dite "horizontale», présidée par Pierre Bourdieu et François Gros et chargée d'assurer la cohérence du travail des précédentes; discussions lors des tables rondes préparatoires à la future loi d'orientation; organisation d'une consultation nationale au moyen d'un questionnaire diffusé à un million d'exemplaires et de colloques académiques; création du Conseil national des programmes (CNP); réforme du statut de l'Inspection générale.

Le diagnostic de crise et les solutions formulés par Lionel Jospin et ses conseillers dans le cadre de ce dispositif participent d'un certain sens commun réformateur qui s'est cristallisé dans l'espace public depuis plusieurs décennies. Leur projet emprunte en effet certains de ses thèmes au mouvement de rénovation pédagogique qui s'est développé dans les années 1960 (d'Enfert \& Kahn, 2011) parallèlement aux réformes de I'organisation scolaire portées par la Ve République gaullienne. Plus encore, et ce malgré les différences de positionnement dans le champ politique, il entretient un lien de filiation très marqué avec les entreprises de modernisation et de rénovation initiées par Joseph Fontanet et René Haby dans les années 1970 (Aebischer, 2010, p. 380-419; Robert, 2010a, 2016). De manière plus attendue, il s'inscrit enfin dans la lignée des initiatives prises par les précédents gouvernements socialistes pour réformer les programmes et la pédagogie, notamment les commissions permanentes de réflexion sur l'enseignement des disciplines mises en place par Alain Savary et les Propositions pour l'enseignement de I'avenir du Collège de France, rédigées sous la conduite de Pierre Bourdieu et remises au président de la République en mai 1985 (Heurdier \& Clément, 2016).

Au-delà de la volonté de mettre en œuvre une réforme coordonnée et simultanée de l'ensemble des disciplines en fonction de principes communs applicables de manière transversale à chacune d'entre elles, la particularité et l'intérêt de cette séquence réformatrice résident dans l'encastrement des questions pédagogiques dans des enjeux budgétaires, statutaires, syndicaux et politiques. On ne peut en effet comprendre les prises de position des différents acteurs sur la réforme des contenus d'enseignement en général et la recomposition des champs disciplinaires en particulier sans prendre en compte les liens très étroits qui existent entre, d'un côté, ces questions proprement lorisation de la rémunération des enseignants, les velléités de réduction des grilles horaires, les projets de refonte des différents corps enseignants, les luttes d'appareil entre les deux principales tendances de la Fédération de l'Éducation nationale (FEN) (voir encadré) et, enfin, les luttes internes au PS entre les trois principaux prétendants à la succession de François Mitterrand que sont Laurent Fabius, Lionel Jospin et Michel Rocard.

Afin d'appréhender les transformations du curriculum formel qui se jouent dans une telle configuration, il a été nécessaire de sortir d'un cadre d'analyse qui prendrait comme échelle et comme objet d'analyse telle ou telle discipline en particulier. En effet, qu'on les considère comme des «corps de savoirs spécialisés, reconnus et transmis comme tels par les organisations universitaires» dotés d'une triple identité «sociale», «professionnelle» et «épistémologique» (Boutier, Passeron \& Revel, 2006), comme des «territoires» occupés par des «tribus académiques» (Becher, 1989), comme des "communautés sociales» (Musgrove, 1968) ou comme des champs (Bourdieu, 1976), les disciplines constituent en tous cas des univers sociaux autonomes et institutionnalisés qui s'organisent autour d'enjeux, à la fois savants et didactiques, d'acteurs et d'institutions sui generis. Du fait de cette autonomie, elles exercent ainsi un puissant effet de réfraction (Bourdieu, 1991, 1997) qui tend à transfigurer, voire à masquer, les déterminants externes pouvant exercer un effet sur la transformation des programmes d'enseignement. Or, étant donné que la séquence historique étudiée ici se caractérise justement par la forte emprise de ces déterminants externes sur les enjeux proprement pédagogiques, il donc fallu faire porter l'enquête ${ }^{1}$ non seulement sur les lieux où peut se jouer la

1 Cet article s'appuie notamment sur le dépouillement des archives du SNI-PEGC, du cabinet de Lionel Jospin et de l'un de ses conseillers, du SNES (fonds Denis Paget), du Parti socialiste (fonds Marie-France Lavarini, collaboratrice de Lionel Jospin lors de son mandat de premier secrétaire du Parti socialiste entre 1981 et 1988) et de Pierre Bourdieu ainsi que sur une série d'entretiens approfondis avec les principaux acteurs de la période (membres des cabinets de Lionel Jospin, Michel Rocard et François Mitterrand, chercheur et historien de l'éducation mobilisé dans le cadre de la consultation nationale organisée par le ministère de l'Éducation nationale, dirigeants syndicaux, directeur des Lycées et collèges, conseillers de Laurent Fabius). Une demande de dérogation a également été effectuée pour consulter le fonds Antoine Prost déposé aux Archives nationales afin de connaître la position et I'action du Premier ministre et de son principal conseiller mais l'accès m'a été refusé s'agissant des documents véritablement intéressants au motif que "le Secrétariat général du gouvernement considère qu'il s'agit de documents portant des appréciations et des jugements de valeur 
Ce paysage est encore nettement dominé par la Fédération de l’Éducation nationale qui peut alors être qualifiée de forteresse enseignante (Aubert, Bergounioux, Martin et al., 1985). Ayant choisi l'autonomie en 1948 après la scission entre la CGT (Confédération générale du travail) et FO (Force ouvrière), la FEN rassemblait plusieurs dizaines de syndicats nationaux et voyait sa vie interne structurée autour de tendances. Héritière du courant ayant fait le choix de l'autonomie en 1948 et «fédérant la gauche républicaine, socialiste, radicale et franc-maçonne» (Geay, 2005), la tendance UID (Unité, indépendance, démocratie) est majoritaire au sein du principal syndicat, le SNI-PEGC (Syndicat national des instituteurs-Professeurs d'enseignement général de collège), et également au niveau fédéral. Héritière du courant ayant soutenu le rattachement à la CGT en 1948 et dirigée par des militants membres ou proches du Parti communiste, la tendance UetA (Unité et Action; ou UA) est majoritaire au sein du SNES (Syndicat national des enseignements de second degré) mais minoritaire au niveau fédéral. En marge de ces deux tendances centrales, on trouve des tendances, comme l'École émancipée, regroupant des militants issus du trotskysme et/ou de l'anarcho-syndicalisme. Moins représentatives, elles sont toutefois très actives dans la vie et les équilibres internes de la Fédération.

Créé en 1937 et d'abord affilié à la CFTC (Confédération française des travailleurs chrétiens) puis à la CFDT (Confédération française démocratique du travail) depuis sa création en 1964, le SGEN (Syndicat général de l'Éducation nationale) est la deuxième organisation la plus importante du paysage syndical enseignant français. Sa particularité est d'être un syndicat intercatégoriel auquel peut adhérer l'ensemble des personnels, de la maternelle à l'Université. Très peu présent dans l'enseignement primaire jusqu'au début des années 1980, il est surtout implanté dans le second degré (et dans l'enseignement supérieur). Renforçant «son image d'organisation critique » après 1968, il apparaît dans les années 1980 comme une sorte de "laboratoire d'idées" de la gauche enseignante, à mi-chemin de la réflexion pédagogique et de la démarche revendicative» (Geay, 2005, p. 10).

À côté de la FEN et du SGEN, un troisième acteur important est apparu au début des années 1980 avec le développement de syndicats enseignants au sein de FO. Créé en 1984 par des militants trotskystes et des militants gaullistes hostiles à la politique de rénovation pédagogique d'Alain Savary, le SNUDI-FO (Syndicat national unifié des directeurs et des instituteurs) va voir son audience rapidement augmenter. Aux élections professionnelles de décembre 1984, le rapport des forces entre ces trois organisations s'établit ainsi : la FEN obtient $58,5 \%$ des suffrages (en baisse de 7,5 points), le SGEN $15 \%$ (en baisse de 1,5 point), FO $12 \%$ (en hausse de 9,5 points) (Geay, 2005, p. 95).

En marge de ces trois organisations dominantes, on trouve quelques syndicats autonomes comme le SNALC (Syndicat national des lycées et collèges), qui se distingue par sa défense du second degré et son conservatisme pédagogique, ou encore le SNC (Syndicat national des collèges), dont la défense des intérêts catégoriels des PEGC constitue la principale raison d'être.

Dans cet article, il sera essentiellement question de la FEN, de ses deux syndicats les plus importants et de ses deux principales tendances. Toute l'enquête archivistique, comme tous les entretiens réalisés, de même que le dépouillement de la presse spécialisée de l'époque montrent de manière concordante que l'ensemble des autres acteurs se trouvent de facto mis sur la touche par Lionel Jospin et son équipe qui ne négocient véritablement qu'avec la FEN, le SNI-PEGC et le SNES.

recomposition des champs disciplinaires, mais aussi sur les différents champs - syndical, bureaucratique et politique en l'occurrence - dans lesquels s'inscrivent les différents acteurs qui sont intéressés à cette recomposition. Puisque les espaces et les configurations d'acteurs dans lesquels se jouent ces tentatives de recomposition changent en fonction de la période considérée, chacune des trois parties de cet article adopte donc une focale singulière.

En revenant sur la genèse et les implications multiples de la création d'un corps de professeurs de col-

sur des personnalités politiques encore vivantes susceptibles de leur causer préjudice». lège, on montrera tout d'abord en quoi la recomposition des champs disciplinaires procède d'un projet corporatif et statutaire, qui procède lui-même des luttes d'appareil entre les deux principales tendances de la FEN. On verra ensuite pourquoi L. Jospin, après I'avoir accepté pendant un cours laps de temps, finit par abandonner l'idée de réformer l'organisation pédagogique du collège en modifiant le statut des enseignants qui y enseignent. On analysera enfin ce qu'il est advenu des tentatives pour donner corps à la recomposition des champs disciplinaires malgré l'abandon de la réforme statutaire. 


\section{Inscrire le collège dans la continuité de l'école primaire : les origines syndicales du corps des professeurs de collège}

En 1988, lorsque L. Jospin entame les négociations sur la revalorisation de la condition enseignante et la future loi d'orientation, les dirigeants de la tendance UID, majoritaire chez les instituteurs et donc à la tête du SNI-PEGC et de la FEN, réclament la création de trois corps dits "comparables». Aux corps exerçant alors dans l'enseignement scolaire - instituteurs, PEGC, certifiés, adjoints d'enseignement, etc. - se substitueraient trois nouveaux corps : professeurs des écoles, professeurs des collèges, professeurs de lycée. Les enseignants recrutés dans ces nouveaux corps le seraient à bac +3 , recevraient ensuite une formation professionnelle différenciée en fonction du niveau d'enseignement choisi et bénéficieraient enfin d'une même grille indiciaire. Amenés à remplacer les PEGC et les professeurs certifiés, les nouveaux professeurs des collèges auraient alors vocation à intervenir sur des champs disciplinaires recomposés et élargis ne correspondant ni aux bivalences des premiers ${ }^{2}$, ni aux spécialités le plus souvent mono-disciplinaires qui sont au cœur de l'identité professionnelle des seconds. En réactivant et en reprenant ainsi l'idée, déjà présente chez les réformateurs des années 1960 puis portée politiquement par les ministres Joseph Fontanet ${ }^{3}$ et René Haby ${ }^{4}$ dans les années 1970, de «constituer un corps enseignant intermédiaire entre la non-spécialisation des instituteurs et la spécialisation des professeurs de lycée» (Prost, 2007, p. 148), il s'agit pour le SNI-PEGC et la FEN, au nom du principe de continuité éducative, de lutter contre la structuration disciplinaire de l'enseignement au collège et pour la réduction du nombre de professeurs auxquels doivent faire face les collégiens, et ainsi d'atténuer, sur les plans statutaire et pédagogique, la rupture entre le collège et l'école primaire. Restructurée autour de ces nouveaux champs disciplinaires, fondée sur des programmes renouvelés

2 Sur le plan pédagogique, la spécificité des PEGC était qu'ils enseignaient deux disciplines scolaires. Par exemple : lettres et langues vivantes, lettres et histoire-géographie, mathématiques et éducation musicale, mathématiques et sciences physiques, etc.

3 Projet de loi portant réforme de l'enseignement du second degré, mars 1974. en conséquence et allégés, assurée par des maîtres polyvalents, la nouvelle organisation pédagogique du collège en ferait dès lors non plus l'antichambre du lycée général, mais bien le prolongement de l'école primaire. À travers ce projet, indissociablement statutaire et pédagogique, de création de corps comparables, se rejoue une des questions structurantes de I'histoire scolaire depuis le milieu du XIX ${ }^{\mathrm{e}}$ siècle (Chapoulie, 2010; Prost, 1968) : celle du positionnement de l'école moyenne dans le système d'enseignement et, partant, des luttes de territoire entre l'univers primaire (Geay, 1994) d'un côté et le "grand second degré» de l'autre (Lelièvre, 2011).

\section{De l'école fondamentale...}

Dans un mouvement similaire à celui qui avait conduit le SNI, à partir de la fin des années 1960, à dépasser le «trouble profond» qui fut d'abord le sien vis-à-vis de la création du corps des PEGC (Robert, 2010b, p. 124), la majorité UID du SNI-PEGC et la FEN vont intégrer la création d'un corps de professeurs de collège à leur stratégie visant à "sauver et ennoblir l'école primaire ${ }^{5}$. Cette stratégie s'enracine dans le contexte du début des années 1960, au moment où plusieurs facteurs, tant institutionnels que sociodémographiques, modifient profondément la place et la fonction de l'univers primaire dans le système scolaire en train de se constituer. Alors que la généralisation de l'enseignement post-élémentaire est déjà bien amorcée et que se pose la question du type d'établissement, de filière et de maître appelé à scolariser l'ensemble des enfants âgés de 12 à 16 ans, l'enseignement primaire doit subir une concurrence de plus en plus vive de la part de l'enseignement secondaire. Celui-ci va en effet capter une part toujours plus importante des élèves poursuivant une scolarisation prolongée. La prolongation de la scolarité obligatoire jusqu'à 16 ans introduite par l'ordonnance du 6 janvier 1959 et ses conséquences, notamment au plan institutionnel, conduisent à l'affaiblir encore davantage en faisant voler en éclat son unité administrative, statutaire et syndicale. La suppression des cours complémentaires, qui étaient des classes administrativement rattachées aux écoles primaires, et

5 Titre de la session générale d'études des 8, 9 et 10 avril 1965 du SNI. Cette conversion à l'idée d'un corps unique de professeurs de collège n'est pas si surprenante lorsqu'on se souvient que le SNI avait vu dans cette disposition Fontanet une ouverture intéressante (Brucy, 2003; Robert, 2016). 
leur remplacement par les Collèges d'enseignement général (CEG), qui sont des établissements autonomes, consacre la coupure administrative entre l'école élémentaire et le premier cycle du second degré. La création du Certificat d'aptitude au professorat des collèges d'enseignement général en $1960^{6}$ qui sépare les «maîtres de CEG » des instituteurs, puis celle du corps des PEGC en 19697, entérinent quant à elles cette coupure sur un plan statutaire. Enfin, la progression de la minorité dite "cégétiste» ou «unitaire» (Frajerman, 2003) et la création en 1960 du Syndicat national des collèges (SNC) (Robert, 2010b) remettent en cause la situation de quasi-monopole de la représentation syndicale qui prévalait alors dans l'univers primaire. Alors que «vers 1930, l'immense majorité des enfants ne fréquentaient pas d'autre école que l'école primaire» et que "pour six enfants sur sept, environ, c'était la seule école» (Prost, 2004, p. 166), le SNI ne peut que constater, dès 1964, que l'enseignement primaire tend peu à peu à se restreindre aux seules classes maternelles et élémentaires : «cessant d'être une fin en soi, [il] va devenir, pour tous les enfants, une étape dans le déroulement de leurs études ${ }^{8}$.

$C^{\prime}$ est pour tenter d'endiguer ce processus et d'empêcher cette fin annoncée de l'univers primaire que la direction du SNI va élaborer le projet dit d'«École fondamentale». Finalisé lors du Congrès de Nantes en 1971 et diffusé largement dans une version enrichie en $1973^{9}$, ce projet éducatif global articule revendications corporatives et pédagogiques autour d'un grand principe : la continuité éducative de la maternelle jusqu'au collège. Il s'agit ainsi d'assurer la défense de l'univers primaire en revendiquant son extension jusqu'à la fin de la scolarité obligatoire. Sur le plan des structures, ce principe se traduit principalement par la volonté de supprimer les filières alors existantes dans les Collèges d'enseignement secondaire (CES). Sur le plan statutaire, ce même principe conduit le SNI à revendiquer la création d'un corps de maîtres de l'École fondamentale bénéficiant d'une formation commune, dispensée

\footnotetext{
6 Décret $n^{\circ} 60-1127$ du 21 octobre 1960.

7 Décret $n^{\circ} 69-493$ du 30 mai 1969.

8 L'École libératrice, $n^{\circ} 39,7$ septembre 1964, p. 1862.

9 L'École libératrice, $\mathrm{n}^{\circ} 26$-bis, 19 mars 1973. Par ailleurs, ce sont 20000 exemplaires d'une brochure d'une centaine de pages qui sont imprimés et diffusés par la maison d'édition SUDEL (Société universitaire d'édition de librairie qui, créée en 1932 par le SNI, édite notamment le «Code Soleil».) Par rapport au projet de 1971, la publication de 1973 est notamment enrichie des résultats de la session d'études de 1972 consacrée à la définition du maître de l'École fondamentale (cf. L'École libératrice, n³2, 8 mai 1972).
}

non pas à l'université mais dans les écoles normales. Polyvalents aux niveaux maternel et élémentaire, ces maîtres de l'École fondamentale seraient appelés à être davantage spécialisés au collège. C'est ainsi que, sur le plan pédagogique, le SNI préconise une recomposition complète des champs disciplinaires afin d'abolir la rupture entre l'enseignement élémentaire dispensé par un seul maître polyvalent et l'enseignement des collèges juxtaposant l'intervention de plusieurs professeurs spécialisés. Le rapport pédagogique du congrès de Nantes de 1971 évoque la possibilité que l'enseignement au collège soit ainsi réorganisé autour de trois «matières de base » - français, mathématiques et langues vivantes -, auxquelles viendraient s'ajouter les activités d'éveil ainsi que l'éducation physique et artistique $^{10}$. S'agissant enfin du contenu même des enseignements, on retrouve dans l'École fondamentale la plupart des principes qui seront au fondement des prises de position du SNI-PEGC en 1988-1989'11 et qui seront ensuite repris par le Syndicat des enseignants (SE-FEN puis SE-UNSA [Union nationale des syndicats autonomes]) au moment de l'élaboration du socle commun de connaissances et de compétences ${ }^{12}$ : rejet de l'encyclopédisme et de l'abstraction, priorité donnée à l'apprentissage de savoir-faire et d'attitudes sur l'acquisition de connaissances factuelles, définition

10 L'École libératrice, $n^{\circ} 37,1^{\text {er }}$ septembre 1971.

11 Cf. la note rédigée pour la réunion de la majorité UID du 19 mars 1986 qui indique que «sous bénéfice d'inventaire, la réflexion pédagogique conduite sur ces vingt dernières années reste valable (finalités, contenus) » ou encore cette prise de position de JeanClaude Barbarant qui, lors du Bureau national du 12 novembre 1987, affirme que «le projet du SNI-PEGC prolonge la logique de l'école fondamentale, en prenant en compte les réalités d'aujourd'hui» (L'École libératrice, $n^{\circ} 11,21$ novembre 1987).

12 Dans un entretien réalisé en avril 2007, Philippe Niemec, secrétaire national du SE-UNSA en charge du secteur Éducation, insiste sur la continuité entre le projet d'École fondamentale et les prises de position de son syndicat lors des débats sur le socle commun : "On parle dans nos mandats, dans les mandats syndicaux, de socle commun depuis bien plus longtemps que 2002. On remonte beaucoup plus loin. Le SNI avait par exemple un projet éducatif, y a une trentaine d'années qui s'appelait le projet de l'école fondamentale. Je sais pas si vous avez entendu parler de ce projet qui a marqué les esprits y compris d'ailleurs des responsables qui sont aujourd'hui des hauts fonctionnaires. J'étais surpris de la part de certains responsables ministériels, d'entendre parler d'école fondamentale, comme quoi ça avait vraiment marqué son temps. Donc l'école fondamentale, c'était bien d'assurer une continuité éducative école-collège, d'abord en faisant en sorte qu'il n'y ait pas de rupture entre les différents niveaux. Et qu'ensuite il y ait une continuité dans les programmes, dans les acquisitions des programmes, en faisant que l'ensemble de ces programmes devaient constituer l'essentiel des savoirs et des compétences à faire acquérir par tous les élèves. Donc c'était ça l'école fondamentale. Donc de fait, on est revenu à cette conception que le syndicat avait par le passé.» 
des programmes sous la forme d'objectifs, orientation pratique et concrète des contenus ouverts sur la vie, nécessité d'une mise à jour continue des savoirs.

\section{... à son aggiornamento dans le milieu des années 1980}

Si l'École fondamentale constitue toujours la matrice idéologique du SNI-PEGC, de la FEN et de la tendance UID en 1988-1989, leur projet syndical a cependant été modifié sur des points essentiels. Confronté à son incapacité à obtenir la mise en œuvre de l'École fondamentale de la part d'un gouvernement dont il est pourtant très proche politiquement, aux premiers signes manifestes du déclin de la syndicalisation et de l'érosion de sa représentativité (Geay, 2005, p.85-98) ainsi qu'à la croissance accélérée de la scolarisation dans le second degré, notamment dans les lycées, où UID n'est pas majoritaire, le SNI-PEGC entame en effet, dès la rentrée 1985, un profond aggiornamento de ses objectifs revendicatifs.

Plus que les revendications pédagogiques de l'École fondamentale, considérées comme globalement acceptées par les enseignants, ce sont bien les revendications statutaires qui posent problème, et notamment l'idée de tendre vers l'unité catégorielle "dans le sens de mêmes enseignants pour l'école fondamentale » ${ }^{13}$. Qu'elle déplore le «complexe rentré du certifié $»^{14}$ ou l'«aspiration élito-catégorielle $»^{15}$ dont seraient victimes les PEGC, la direction du SNI-PEGC doit en effet admettre que faire passer la promotion des PEGC par l'intégration dans un corps de maîtres de I'École fondamentale ne peut constituer une réponse syndicale adaptée aux aspirations de cette catégorie d'enseignants. Cela reviendrait en effet à les assimiler aux instituteurs alors même qu'ils ont les yeux tournés vers l'enseignement secondaire. Aussi, et tandis que «l'augmentation forte prévisible pour les postes de

13 «Document récapitulatif pour les trois regroupements UID du $1^{\text {er }}$ trimestre " joint à une lettre de Brigitte Fillon aux secrétaires de section UID du 29 janvier 1986. Archives du SNI-PEGC, boîte $n^{\circ} 1526$. 14 Ibid.

15 «Note de réflexion pour la réunion majoritaire du 19 mars 1986 ». Archives du SNI-PEGC, boîte n ${ }^{\circ} 1526$. L'auteur de cette note est inconnu. Toutefois Hervé Baro, alors secrétaire académique du SNI-PEGC et membre du bureau national et auquel j'ai soumis cette note lors d'un entretien, m'a indiqué que seules deux personnes pouvaient l'avoir produite : Claude Lavy, secrétaire national à la vie interne, et Luc Bentz, secrétaire départemental du SNI-PEGC Paris. Étant donné qu'elle est jointe à un courrier envoyé par C. Lavy, on peut penser que c'est ce dernier qui en est l'auteur. second degré long accroît le potentiel de syndicalisa-

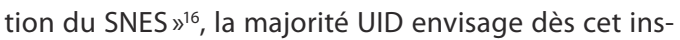
tant une remise en cause prochaine des équilibres internes à la FEN. Celle-ci pourrait aboutir à «la mainmise de l'appareil UA du SNES, c'est-à-dire du PC [Parti communiste], sur le grand second degré et par ce biais (avec la massification) sur la FEN $\aleph^{17}$. Adoptant une tonalité volontiers dramatique, un membre du secrétariat national du SNI-PEGC affirme que «le syndicat est entré dans une phase charnière de son histoire » : il insiste dès lors sur la «nécessité absolue de réajuster nos objectifs » et de mener « une réflexion sans tabou», n'excluant $a$ priori aucune hypothèse, "fût-elle sacrilège » et «même si elle met en cause nos choix antérieurs $»^{18}$. Ainsi, tout en reconnaissant que "c'est sans doute ce que préconise le SNC et que nous avons combattu», mais considérant «qu'il s'agit ici et maintenant de savoir si nous voulons nous donner ou non les moyens de combattre victorieusement la mainmise des conservateurs, des élitistes et des communistes (objectivement alliés en l'occurrence) sur le collège, si nous renonçons ou non à nos objectifs de démocratisation », en vient-il à envisager la création d'un "corps de prof de collège intégrant les actuels certifiés [...] et les actuels PEGC »'

Alors que la tendance UID a déjà commencé à s'interroger sur la redéfinition de ses objectifs corporatifs et de sa stratégie syndicale, l'alternance politique de 1986 va la conduire à précipiter considérablement le processus qu'elle avait amorcé au début de l'année scolaire 1985. Issu de la démocratie chrétienne, René Monory, le nouveau ministre de l'Éducation nationale du gouvernement de cohabitation d'Édouard Balladur, décide en effet, le 28 avril 1986, de mettre un terme aux recrutements dans le corps des PEGC. Dorénavant, seuls les professeurs certifiés et agrégés auront donc vocation à enseigner dans les collèges. Dès lors, l'éviction du SNI-PEGC et d'UID des collèges ainsi que la prise de pouvoir de la minorité UA au sein de la FEN deviennent une perspective de court terme à laquelle il faut réagir rapidement. Cette réaction va s'échelonner en trois temps. Les dirigeants d'UID vont en effet s'accorder tout d'abord sur une refonte radicale des structures de la FEN, puis sur une redéfinition majeure de leurs objectifs corporatifs et statutaires et, in fine,

\footnotetext{
16 Ibid.

17 Ibid.

18 Ibid.

19 Ibid.
} 
sur une adaptation de leur projet pédagogique à cette nouvelle stratégie. Dès le début du mois de mai 1986, soit quelques jours à peine après la décision de René Monory, les nombreuses notes qui circulent au sein des dirigeants de la majorité UID se focalisent en effet sur les différents scénarios d'une possible recomposition syndicale et d'une modification des champs de syndicalisation à l'intérieur de la FEN. Approfondie à la rentrée 1986 lors d'une série de réunions de tendance, cette réflexion conduit la majorité UID à retenir l'idée de créer, au sein d'une FEN élargie, un Syndicat général des enseignants de la maternelle aux portes de l'enseignement supérieur ${ }^{20}$. Cette décision apparaît en effet comme la «seule de nature à conserver à la FEN son orientation d'aujourd'hui $»^{21}$. Sont ainsi écartées la solution consistant à créer un syndicat de l'École fondamentale, qui aurait regroupé les instituteurs et tous les enseignants du collège, et celle consistant à créer deux syndicats distincts : I'un pour les enseignants des écoles, maternelles et élémentaires, l'autre pour les enseignants des collèges.

Considérant qu'«il est bien évident que ce syndicat général devra définir un projet éducatif revendicatif et corporatif qui devra assurer sa cohésion $»^{22}$, les dirigeants d'UID vont ensuite s'attacher à redéfinir le cadre de leurs revendications statutaires. Déjà évoquée avant la décision de René Monory, la création de trois corps dits comparables est finalement actée en interne en avril-mai $1987^{23}$ et inscrite dans la motion revendicative votée au congrès du SNI-PEGC qui se tient à Lille en juin $1987^{24}$. Revendication historique du SNI-PEGC et clé de voûte du mythe de l'école unique, la création d'un corps unique d'enseignants est ainsi abandonnée au motif qu'elle serait ingérable d'un point de vue administratif.

Si la décision de René Monory a donc conduit le SNI-PEGC et sa majorité UID à faire évoluer de manière significative leurs stratégies d'appareil et leurs revendications corporatives, il n'en va pas de même sur le plan pédagogique. Dans une note interne, un membre du bureau national écrit ainsi au sujet du projet édu-

20 Comme le montrent les notes échangées au sein de la direction du SNI-PEGC, cette solution est actée dès le mois de janvier 1987. 21 "Note de réflexion sur l'avenir du SNI-PEGC et de la FEN", note d'H. Baro à J.-C. Barbarant, 10 mai 1986. Archives du SNI-PEGC, boîte $\mathrm{n}^{\circ} 1526$

22 Ibid.

23 «Propositions pour le débat revendicatif - Lille 87 ». Archives du SNI-PEGC, boîte n' 1526.

24 L'École libératrice, n², 12 septembre 1987. catif qui devra être celui du futur syndicat général : «au plan éducatif, l'école fondamentale et le projet éducatif de la FEN fournissent une bonne base de départ qu'il ne sera sans doute pas utile de modifier de façon conséquente $»^{25}$. De même, dans la motion éducation du congrès de Lille, le SNI-PEGC se contente de réaffirmer «l'actualité des revendications du syndicat en matière de contenus d'enseignement $»^{26}$. Sur le plan éducatif et pédagogique, l'aggiornamento de l'École fondamentale ne va s'opérer que dans un troisième temps, à la faveur du rapprochement entre le nouveau secrétariat national à l'éducation du PS, dirigé par L. Fabius à partir d'avril 1987, et la direction de la FEN, au moment où celle-ci entreprend de préparer son congrès de La Rochelle prévu pour le début de l'année 1988.

\section{Un projet négocié entre la FEN et le PS}

Dans l'éventualité d'une possible alternance politique, les deux organisations vont alors mener pendant plusieurs mois des négociations pour finalement parvenir à un accord donnant-donnant : « revaloriser » la condition enseignante en échange d'un engagement à « travailler autrement ", c'est-à-dire non seulement moderniser le fonctionnement du système éducatif, mais aussi rénover les pratiques pédagogiques, modifier les contenus d'enseignement et transformer le métier de professeur.

Au-delà de la proximité politique entre la direction de la FEN et le secrétariat à l'éducation du PS, cet accord repose sur un diagnostic partagé quant aux causes des dysfonctionnements de l'Éducation nationale. À l'instar des dirigeants de la FEN, L. Fabius ${ }^{27}$ et ses conseillers - notamment Christian Bècle, Christian Forestier, Bernard Pêcheur et Yvon Robert - voient dans «l'élitisme républicain » I'un des principaux obstacles à la démocratisation de l'enseignement ${ }^{28}$ car, selon eux, il

25 L'École libératrice, $n^{\circ}$ 2, 12 septembre 1987.

26 "Note de réflexion sur l'avenir du SNI-PEGC et de la FEN", note d'H. Baro à J.-C. Barbarant, 10 mai 1986. Archives du SNI-PEGC, boîte $\mathrm{n}^{\circ} 1526$.

27 Secrétaire national du Parti socialiste en charge de l'éducation depuis le mois d'avril 1987 et député de la Seine-Maritime depuis le mois d'avril 1986, Laurent Fabius deviendra président de l'Assemblée nationale après l'alternance de 1988.

28 Entretien avec Christian Bècle, à son domicile, Neuilly-sur-Seine, 9 février 2010. Les préventions de Laurent Fabius vis-à-vis des orientations politiques de son ministre de l'Éducation nationale sont par ailleurs évoquées dans un article paru le vendredi 4 janvier 1985 du Nouvel Observateur intitulé "École : le retour à l'ordre», dont I'auteur rapporte l'épisode suivant : «Il y a quelque temps, Laurent 
conduirait à concevoir l'ensemble du système scolaire en fonction des exigences des classes préparatoires et des grandes écoles, et non en fonction des besoins des élèves les plus défavorisés. Dès lors, la réorganisation pédagogique du second degré et la redéfinition du métier de professeur apparaissent comme deux chantiers prioritaires. Dans cette perspective, la promotion d'une conception alternative du métier d'enseignant apparaît comme le principal levier de transformation du système d'enseignement. «Partisans du prof éducateur dans son établissement», contrairement au SNES décrit comme "partisan du prof disciplinaire» et pour qui «la discipline passe avant tout $»^{29}$, Laurent Fabius et ses conseillers PS vont donc envisager une modification du statut des enseignants du second degré consistant, d'une part, à annualiser leur temps de travail et, d'autre part, à intégrer à leurs obligations de service des tâches autres que l'enseignement devant une classe, ce qui reviendrait à porter la durée hebdomadaire de leur présence dans l'établissement à 35 heures. Déclinée sur le plan pédagogique, cette conception du "prof éducateur » se traduirait notamment par la mise en place de la bivalence des enseignants de collège et le redécoupage des champs disciplinaires, deux mesures qui font directement écho aux prises de position défendues par le SNI-PEGC depuis l'élaboration de l'École fondamentale.

Conduites sur la base de cette forte convergence idéologique, les négociations entre le PS et la FEN débouchent finalement sur un accord de principe : tandis que le premier s'engage sur la création de corps comparables recrutés au niveau licence et rémunérés sur la grille indiciaire des certifiés, la seconde s'engage quant à elle sur quatre contreparties : annualiser le temps de service, intégrer des tâches pédagogiques nouvelles dans les obligations de service, introduire de nouvelles modalités de gestion des carrières et de l'avancement, donner enfin plus de pouvoir aux familles dans le processus d'orientation ${ }^{30}$.

Conçue à l'origine par la majorité UID de la FEN comme un moyen de perpétuer sa domination dans le

Fabius aborde Jean-Pierre Chevènement qui discute avec une dizaine de recteurs : "Comment expliquez-vous, monsieur le Ministre, que votre politique soit soutenue par nos ennemis et combattue par nos amis?" Un ange est passé. Et repassé car Fabius, quelques jours plus tard, a repris le même thème lors d'une réunion où le ministre était face à tous les recteurs d'académie de France.» 29 lbid.

30 «La revalorisation de la carrière des enseignants. Entrevue avec champ syndical, la réforme du statut des enseignants devient, aux termes de cet accord, un levier essentiel de la réforme pédagogique. Aussi, pour sceller publiquement son engagement, la FEN adopte-t-elle, lors de son congrès de La Rochelle de février 1988, un nouveau projet éducatif, «l'École de l'an 2000 », qui se substitue non seulement à son ancien projet d'«École de l'éducation permanente », élaboré dès les années 1970 et finalement adopté en 1981 (Brucy, 2003), mais aussi, et surtout, à l'École fondamentale. Négociée lors d'une dernière réunion au sommet entre la FEN et le secrétariat national du PS ${ }^{31}$, la formule «travailler autrement» va s'imposer comme le mot d'ordre appelé à symboliser et à synthétiser l'ensemble des propositions de rénovation du système éducatif portées publiquement par la FEN pour «l'École de l'an 2000 » : continuité éducative désormais revendiquée jusqu'aux portes de l'enseignement supérieur et conçue comme un prélude à une éducation tout au long de la vie, pédagogie différenciée pour répondre à l'hétérogénéité des élèves, respect de la diversité des rythmes d'apprentissage, refus de l'émiettement et de la juxtaposition disciplinaire au collège, lutte contre l'encyclopédisme et la surcharge des programmes, rédaction des programmes sous la forme de référentiels de savoirs et de savoir-faire, révision périodique des contenus d'enseignement, évaluation formative qui ferait du diplôme la sanction de compétences effectives, travail en équipe, etc. ${ }^{32}$

À la veille de l'élection présidentielle de 1988 , le SNI-PEGC et la FEN ont donc énoncé les principes qui constituent l'assise pédagogique de leur projet de créer trois corps comparables. II reste cependant à leur donner une traduction concrète. Alors que la transformation de l'organisation pédagogique du collège apparaît comme une priorité, ses modalités demeurent en effet indéterminées. C'est le cas notamment de la recomposition des champs disciplinaires que présuppose la création du corps de professeurs de collège et qui va s'imposer comme l'un des principaux enjeux des luttes scolaires après l'arrivée de L. Jospin au ministère de l'Éducation nationale.

31 Cette réunion a pris la forme d'un petit-déjeuner organisé à I'hôtel Sofitel-Bourbon le $1^{\text {er }}$ février 1988, c'est-à-dire le matin même de l'ouverture du congrès de la FEN. Étaient présents pour le PS, L. Fabius, C. Bècle, Bernard Pêcheur et Michel Delebarre - secrétaire national chargé des relations avec les syndicats; pour la FEN, Jacques Pommatau - ancien secrétaire général - et Yannick Simbron - secrétaire général; pour le SNI-PEGC, J.-C. Barbarant.

32 L'Enseignement public, supplément au n 50, avril-mai 1988. 


\section{Réformer le statut des enseignants pour transformer l'organisation pédagogique du collège : un projet mort-né}

\section{L'échec d'un scénario ou le refus du donnant-donnant par Lionel Jospin}

La réélection de F. Mitterrand accouche d'une configuration politique qui vient bouleverser le scénario envisagé par la majorité UID de la FEN et le secrétariat à l'éducation du PS. Tandis que Michel Rocard devient Premier ministre, L. Jospin se retrouve, au gré des arbitrages auxquels donne lieu la composition d'un gouvernement, nommé à la tête d'un grand ministère regroupant l'Éducation nationale, la Recherche et les Sports. Dès le lendemain de sa nomination, le secrétaire général de la FEN, Yannick Simbron, lui adresse une demande d'audience ${ }^{33}$, laquelle se tient finalement le 17 mai 1988. Celle-ci est notamment l'occasion de signifier à L. Jospin que le travail effectué avec l'équipe de L. Fabius «ne devrait pas être perdu » ${ }^{34}$. Signe de l'importance de l'enjeu, l'accord négocié entre la direction de la FEN et l'équipe de Laurent Fabius est au cœur des premières discussions qui s'établissent alors entre la FEN et l'entourage du ministre. Dans la perspective d'une nouvelle audience prévue dès le 30 mai 1988, le directeur de cabinet adjoint de L. Jospin lui fait ainsi savoir que «la FEN souhaite voir le Ministre d'État en accord avec le précédent premier secrétaire [c'est-àdire Lionel Jospin lui-même]» : puisque «des objectifs éducatifs avaient été énoncés parallèlement par le PS et par la FEN, [et qu'] ils convergeaient sur un grand nombre de points, la FEN souhaite s'entendre dire qu'ils demeurent d'actualité $»^{35}$. Or le ministre va refuser catégoriquement $d$ 'honorer les engagements pris par le secrétariat national de son propre parti.

Cette décision tient d'abord à des conflits internes au champ politique. Apparue au grand jour à l'occasion de la campagne pour les élections législatives de 1985, la rivalité entre L. Jospin et L. Fabius est alors particulièrement vive, au moment où se profile déjà la guerre de succession de F. Mitterrand qui éclatera au congrès

33 Lettre de Y. Simbron à L. Jospin, 13 mai 1988. AN, 20040313, art. 1

34 Notes manuscrites de L. Jospin. AN, 20040313, art. 1.

35 Note de Pierre Trincal à L. Jospin, 27 mai 1988. AN, 20040313, art.3. de Rennes en 1990. L'élection du premier secrétaire du PS en mai 1988 constitue le premier terrain de l'affrontement des deux hommes. En soutenant Pierre Mauroy, L. Jospin parvient à faire échec à la candidature de L. Fabius, qui bénéficie pourtant du soutien de F. Mitterrand. Cette rivalité se rejoue ensuite dans les nominations des entourages ministériels. Les conseillers de L. Fabius sont ainsi écartés de l'équipe qui se constitue alors autour de L. Jospin. Tandis qu'Yvon Robert et Bernard Pêcheur rejoignent respectivement les cabinets du Premier ministre Michel Rocard et du président de la République, Christian Bècle se voit opposer une fin de non-recevoir lorsqu'il fait part à L. Jospin de son souhait de «travailler à la mise en œuvre du projet [négocié avec la FEN] » ${ }^{36}$. Selon C. Bècle, le ministre lui aurait alors répondu : «je ne veux pas que par ton intermédiaire mon succès puisse être attribué à Laurent Fabius ${ }^{37}$. Mais en rejetant l'accord passé avec la FEN, il ne s'agit pas seulement pour L. Jospin de ne pas favoriser un rival. Alors qu'il n'a pas exercé de fonctions ministérielles lors du premier septennat de F. Mitterrand et qu'il a fait toute sa carrière politique à la direction du PS, ce choix est aussi un moyen de se défaire de son image d'homme d'appareil et d'apparaître comme un véritable «homme d'État » en capacité d'incarner l'intérêt général.

Si les enjeux propres au champ politique ont donc pesé lourd dans la décision de L. Jospin, on ne la comprendrait cependant pas sans voir ce qu'elle doit à l'évaluation des rapports de force dans le champ syndical. De leurs premières discussions avec le SNES, les conseillers de L. Jospin ressortent en effet convaincus du fait que celui-ci fera tout pour s'opposer à la redéfinition des obligations de service défendue par la FEN. Craignant les capacités de mobilisation d'un syndicat qu'ils perçoivent comme corporatiste et n'hésitant pas à «jouer à fond la carte catégorielle ${ }^{38}$, ils aboutissent à la conclusion qu'il n'est ni souhaitable ni possible de conditionner la revalorisation du métier à la redéfinition du statut des enseignants ${ }^{39}$.

36 Notes manuscrites de L. Jospin, 21 mai 1988. AN, 20040313, art. 1.

37 Entretien avec $C$. Bècle.

38 Note de Luc Soubré à L. Jospin, 6 juin 1988. AN, 20040313, art. 1.

39 Ibid. et Note de Michel Braunstein à L. Jospin, 6 juin 1988. AN, 20040313, art. 1. Cette position est également défendue par le conseiller spécial de L. Jospin, C. Allègre, qui déclare lors d'un séminaire interne du cabinet: "Je ne suis pas pour le "donnant-donnant", en tout cas pas pour le "travaillez plus, on revalorisera"»; notes manuscrites de L. Jospin. AN, 20040313, art. 1. 
Mais au-delà de la stratégie du «donnant-donnant ", c'est bien le cœur de l'accord conclu entre la FEN et le PS qui va être rejeté par L. Jospin et ses conseillers. Ainsi, dès le mois d'août, L. Jospin fait part au Premier ministre de ses «réticences à l'égard du schéma des corps comparables $»^{40}$. Étudiée de manière approfondie lors de discussions officieuses avec la FEN en septembre 1988, l'instauration d'un même niveau de recrutement et d'un même niveau de rémunération pour les enseignants des écoles, des collèges et des lycées rencontre en effet une opposition ferme de la part du ministre lui-même. Plus que les difficultés gestionnaires et le coût budgétaire d'une telle réforme, ce sont bien des considérations politiques qui expliquent sa position. En marge d'une note que lui adresse son directeur de cabinet au sujet des corps comparables, il écrit en effet : "Non. Ce ne sera accepté ni par les enseignants du second degré, ni par l'opinion ( $\downarrow$ du niveau) $»^{41}$.

\section{S'affranchir des disciplines sur le plan statutaire et pédagogique}

Transmises au cabinet du Premier ministre le 5 décembre 1988, amendées lors de négociations interministérielles et rendues publiques les 17 et 18 janvier 1989 lors de deux tables rondes regroupant près de 40 organisations représentatives, les premières propositions du ministre de l'Éducation nationale ne reprennent donc pas le principe d'un même niveau de recrutement et d'un même niveau de rémunération pour les instituteurs et les professeurs du second degré. Si L. Jospin tourne donc le dos à une revendication essentielle du SNI-PEGC et de la FEN, il leur donne néanmoins satisfaction sur un aspect décisif de leur stratégie : il annonce en effet la création d'un corps de professeurs de collège ${ }^{42}$. Recrutés et rémunérés au même niveau que les professeurs de lycée ${ }^{43}$,

40 Chronologie d'Olivier Schrameck adressée à L. Jospin et reprenant l'enchaînement des rencontres et des étapes de la négociation sur la revalorisation du 23 août 1988 au 18 janvier 1989. AN, 20040313, art. 2

41 «Entretiens avec la FEN au cours du mois de septembre», note d'Olivier Schrameck à L. Jospin, 6 octobre 1988. AN, 20040313, art. 3. 42 «Table ronde sur le projet de loi d'orientation. Intervention de Monsieur L. Jospin», 17 janvier 1989. Archives du SNI-PEGC, boîte $\mathrm{n}^{\circ} 690$.

43 Comme on le trouve parfois écrit dans les notes qui circulent au sein du cabinet, cela revient bien à créer des corps comparables, mais, la nuance est de taille, dans le second degré et dans le second degré seulement. ils seraient en revanche amenés à intervenir sur des champs disciplinaires recomposés ne correspondant pas aux bivalences des PEGC.

Âprement débattue au sein du cabinet de L. Jospin, cette création d'un corps de professeurs de collège y est notamment portée par C. Allègre, lequel a su user de sa proximité personnelle avec L. Jospin pour l'imposer. C'est ce que confirme notamment le témoignage de Jean-Claude Barbarant ${ }^{44}$ recueilli par Sylvie Aebischer : «ll était d'accord avec nous sur l'idée qu'il fallait un corps de professeurs de collège, parce que le collège, c'est pas le lycée. Jospin ne voulait pas mais Allègre y croyait. Comme Allègre est conseiller spécial de Jospin, ça avait un certain poids. On a réussi, grâce à Allègre, à obtenir ce corps de collège ${ }^{45}$. Au-delà de ce témoignage oral, l'attribution de la paternité du corps de professeurs de collège à $C$. Allègre est confirmée par une note de Paul Rollin, conseiller de L. Jospin, qui écrit : "en ce qui concerne les corps des enseignants du secondaire, je vous ai remis ce matin une note sur les professeurs de collège. C. Allègre m'a dit qu'il en ferait une autre, estimant qu'il lui revient de la rédiger étant le créateur de ce nouveau corps, ce que je comprends fort bien ${ }^{46}$. Mieux, cette paternité est revendiquée par $C$. Allègre lui-même quelques années plus tard, en mars 1996, lors d'une longue audition devant le CNP alors présidé par Luc Ferry. Invité à faire part de ses propositions sur la réforme des programmes, l'ancien conseiller spécial de L. Jospin déclare ainsi : «La clé, c'est le collège. [...] Voilà comment moi je vois les choses : je pense qu'il faut qu'il y ait dans la sphère scientifique un professeur de maths et un professeur de sciences [...]. Vous savez quand même - je ne vais pas trahir un secret historique parce que maintenant je crois que c'est connu - que dans la première année du ministère Jospin j'avais fabriqué quelque chose pour le collège. Et puis... Jospin a beau être mon meilleur ami, il m'a lâché en rase campagne, un après-midi sur le collège, en cédant aux pressions du SNES. [...] Je garde un mauvais souvenir de cette affaire, car je crois que c'est le cœur. II faut en venir à des idées simples. Quand il est à l'école, l'enfant a une seule personne en face de lui. II ne faut pas qu'il passe

\footnotetext{
44 Secrétaire général du SNI-PEGC.

45 Entretien avec J.-C. Barbarant réalisé par Sylvie Aebischer que je ne saurais assez remercier pour m'avoir transmis plusieurs des entretiens qu'elle a réalisés pour sa propre thèse.

46 "Négociations concernant la formation des maîtres et les corps des enseignants ", note de Paul Rollin à L. Jospin, sans date. AN, 20040313, art. 3.
} 
à huit professeurs quand il entre au collège, mais à deux ou trois ${ }^{47}$.

Pour C. Allègre, la création d'un corps de professeurs de collège n'est que la traduction logique, sur un plan statutaire, d'une réforme d'ensemble du système d'enseignement dont l'allègement des programmes et la réduction des horaires constitueraient la clé de voûte ${ }^{48}$. Dans cette perspective, où apprendre mieux c'est apprendre moins, il s'agit, au niveau du collège, de s'affranchir du cadre disciplinaire calqué sur le lycée pour confier l'enseignement, non plus à des professeurs spécialisés dans une discipline telle que la définissent le CAPES (Certificat d'aptitude au professorat de l'enseignement du second degré) ou l'agrégation, mais bien à des enseignants compétents, au sens technique et juridique du terme, dans un champ disciplinaire plus large. Reprenant les principes, chers au SNI-PEGC et à la FEN, de la continuité éducative et de la spécificité de l'enseignement au collège, à la fois différent de celui de l'école élémentaire et de celui du lycée, $C$. Allègre préconise donc d'entreprendre une profonde recomposition des champs disciplinaires «de nature tout à fait différente de l'association de deux matières dans les bivalences qui caractérisent les PEGC $»^{49}$. Ainsi «la formation des professeurs de collège porterait sur l'ensemble mathématiques-informatique dont les liens sont évidents ${ }^{50}$. De même «seraient définis des professeurs de "sciences expérimentales" ayant une formation leur permettant d'expliquer les différents états de la matière et de la nature, les différents niveaux d'organisation et de classification», tandis que «les professeurs d'histoire-géographie et sciences humaines auraient en fait la charge de l'enseignement des sciences de I'homme, des sociétés et des civilisations où l'Histoire et la Géographie seraient des trames de présentation et de lecture plus qu'une finalité d'érudition $»^{51}$.

Comme le revendiquent le SNI-PEGC et la FEN, le projet de créer ce nouveau corps revient donc bel et bien à affirmer la spécificité du collège non seulement sur le plan pédagogique, mais aussi sur le plan statutaire.

47 Intervention de C. Allègre devant le CNP, 19 mars 1996. AN, 20070019 , art. 5 .

48 «Faire du neuf avec du vieux. Un programme pour adapter notre éducation nationale aux temps futurs », note de $C$. Allègre à L. Jospin. AN, 20040313, art. 2. Ce document n'est pas daté, mais plusieurs indices permettent de le situer dans la deuxième moitié du mois de septembre 1988.

49 Note sur le corps de professeurs de collège. AN, 20040313, art. 3 .

50 lbid.

51 lbid.
Présenté, non sans raison, dans les médias comme le «principal changement» introduit par la future loi d'orientation $^{52}$, ce projet va pourtant être abandonné quinze jours à peine après avoir été rendu public.

\section{L'abandon du corps des professeurs de collège : le désencastrement du statutaire et du pédagogique}

Le revirement brutal du ministre de l'Éducation nationale tient d'abord aux vives oppositions que ses propositions suscitent dans le champ syndical. Alors qu'il s'était efforcé d'élaborer une solution de compromis ménageant les intérêts des uns et des autres, il doit en effet faire face à l'hostilité croisée du SNI-PEGC et de la FEN d'un côté et du SNES de l'autre. Deux jours seulement après les annonces faites à l'occasion des tables rondes des 17 et 18 janvier, le SNES s'engage ainsi dans une épreuve de force avec le ministère. Vent debout non seulement contre le montant, jugé insuffisant, de l'enveloppe financière dédiée à la revalorisation, mais aussi contre les modalités de cette revalorisation qui privilégie l'octroi de primes à la hausse des indices de rémunération, il annonce une série d'actions dures appelées à se prolonger tout au long des négociations avec le ministère qu'il maintient ainsi sous une pression constante ${ }^{53}$. Du côté du SNI-PEGC et de la FEN, la réaction n'est certes pas totalement hostile. Sans surprise, la création du corps des professeurs de collège est bien accueillie. Dans un article du numéro de L'École libératrice qui paraît le 21 janvier 1989, le SNI-PEGC approuve la «remise en cause de la conception disciplinaire de l'enseignement au collège » et la définition de «champs disciplinaires larges", afin que les enseignants puissent «aider les élèves [...] à opérer les synthèses qu'un élève de cet âge est incapable de faire seul $»^{54}$. Le volet statutaire des propositions du ministre suscite en revanche les plus vives oppositions : dans son éditorial, J.-C. Barbarant affirme qu'«il est intolérable et injustifié de pérenniserlacassureentreinstituteurs et professeurs $»^{55}$,

52 Journal de 20 heures d'Antenne 2 du 17 janvier 1989 (cf. la vidéo disponible sur le site de I'INA. Le passage en question est à 16 minutes et 18 secondes).

537 janvier : première journée nationale de grève "active » et manifestations. 28 janvier : opérations portes ouvertes, rencontres avec les parents, information sur les marchés. 6 février : reconduction de la grève active pour les zones 2 et 3.17 février : reconduction de la grève active pour la zone 1.4 mars : journée nationale de manifestations.

54 L'École libératrice, n¹5, 21 janvier 1989.

55 Ibid. 
tandis que Y. Simbron indique au ministre que ses «propositions [...], au milieu du gué concernant les instituteurs, sont en l'état inacceptables $»^{56}$. Ayant joué jusqu'ici joué la carte de la négociation avec le ministre, le SNI-PEGC et la FEN vont se résoudre à changer de stratégie. Face au mécontentement croissant des enseignants du primaire, dont les échos remontent de la base via les sections départementales ${ }^{57}$, la direction nationale du SNI-PEGC appelle ses adhérents à participer à une manifestation nationale organisée à Paris le $1^{\text {er }}$ février conjointement avec le SGEN-CFDT (Syndicat général de l'Éducation nationale-Confédération française démocratique du travail).

Confronté à un mouvement de protestation qui s'installe et s'amplifie, le ministre va lui aussi changer rapidement de stratégie. Dès le 24 janvier, I'un de ses conseillers se demande "comment faire évoluer les orientations sans prêter le flanc aux critiques d'abandon d'une stratégie présentée comme mûrement réfléchie $~^{58}$. Pour sortir de l'ornière, il suggère au ministre d'adopter une stratégie en deux temps. II s'agirait tout d'abord de réunir rapidement les présidents de la commission dite «horizontale » et des commissions dites «verticales» ou «thématiques» mentionnées dans l'introduction et dont il sera question dans la troisième partie, afin qu'ils apportent leur caution scientifique à la recomposition des champs disciplinaires. Fort de ce soutien, le ministre pourrait ensuite "voir les syndicats pour leur dire que cette recomposition des champs disciplinaires est un objectif incontournable» tout en laissant entendre qu'«[il s'interroge] en revanche sur la nécessité de créer un corps collège», au motif qu'«après tout, on peut très bien redéfinir les compétences des certifiés pour qu'ils répondent à ces nouvelles aires disciplinaires ${ }^{59}$. Cette note esquisse donc un revirement décisif : elle préconise en effet d'abandonner un aspect essentiel du volet statutaire de la réforme tout en réaffirmant la validité de son volet pédagogique. La rénovation des contenus

56 «Table ronde sur la revalorisation. Intervention de Yannick Simbron, secrétaire général de la FEN», 18 janvier 1989. Archives du SNI-PEGC, boîte ${ }^{\circ} 681$.

57 À propos des courriers reçus des sections, on trouve le constat suivant : "C'est une avalanche qui va de l'injure à la condamnation de ce que nous avons fait (dès le 19 janvier) donc avant de savoir comment le syndicat allait réagir». Note de Martine Le Gal aux secrétaires permanents et aux conseillers techniques, 31 janvier 1989. Archives du SNI-PEGC, boîte $n^{\circ} 681$.

58 Note manuscrite au ministre, sans auteur, 24 janvier 1989. AN, 20040313, art. 2 d'enseignement aurait dès lors vocation à devenir le principal levier de transformation du système éducatif et du rapport pédagogique. C'est précisément ce même argumentaire que développe Pierre Moscovici, conseiller technique au cabinet du ministre de l'Éducation nationale chargé des questions budgétaires, quand il écrit, dans une note à L. Jospin destinée à préparer une conférence de presse sur la revalorisation, que «le débat s'est focalisé, à l'excès, sur le corps de collèges » et qu'il faut dès lors «le ramener à l'essentiel » en affirmant publiquement que «le pédagogique l'emporte sur le statutaire $»^{60}$. S'inscrivant encore dans la perspective de sauver le corps de collège aux yeux de l'opinion et des syndicats, Pierre Moscovici suggère d'insister sur le fait que «c'est bien l'analyse, confortée par les travaux des commissions disciplinaires et de la commission transversale (BOURDIEU-GROS), de la spécificité du collège reposant sur des contenus, des modalités particulières d'enseignement, qui a guidé le choix de corps différents mais comparables ${ }^{61}$. Trois jours plus tard, Michel Braunstein, conseiller technique en charge des personnels, se prononce expressément pour l'abandon du corps de collège au motif que cette solution comporte des «inconvénients majeurs » pour des «avantages surestimés ${ }^{62}$. Tout en soulignant sa «gestion extraordinairement complexe ${ }^{63}$ du point de vue administratif, il formule également des objections pédagogiques. Ainsi, la création de ce nouveau corps aboutirait à ce que « 3 catégories de personnels assurant des disciplines différentes» soient amenées à cohabiter dans un même établissement : les anciens PEGC bivalents, les anciens certifiés monovalents et les nouveaux professeurs de collège exerçant sur les nouveaux champs disciplinaires recomposés ${ }^{64}$.

Critiqué d'un point de vue administratif et pédagogique, le corps de professeurs de collège l'est aussi d'un point de vue politique. Soulignant les «risques d'incompréhension de l'opinion», Michel Braunstein explique, d'une part, que «le débat public qui était

60 «Point de presse sur la revalorisation de la condition enseignante», note de Pierre Moscovici à L. Jospin, 24 janvier 1989. AN, 20040313, art. 3.

61 Ibid.

62 "Le corps des professeurs de collège", note de Michel Braunstein à L. Jospin, 27 janvier 1989. AN, 20040313, art. 3.

63 lbid.

$64 \mathrm{lbid}$. La solution consistant à demander, dès la mise en application de la réforme, à tous les enseignants des collèges d'exercer sur les nouveaux champs disciplinaires est impossible selon Michel Braunstein car elle demanderait un effort en termes de formation continue auquel le ministère ne serait pas en mesure de répondre. 
apparu à propos des PEGC risque de resurgir, quelles que soient les précautions qui seront prises pour la justification de la création de ce corps » et, d'autre part, que «la virulente opposition d'un nombre important d'organisations d'enseignants (SNES, SNALC, FO, société des agrégés) entretiendra un climat détestable ${ }^{65}$. Revenant alors sur les raisons qui ont pu conduire le ministère à proposer la création d'un tel corps, il lui donne finalement le coup de grâce en remettant en question l'idée de la spécificité pédagogique du collège. En effet, selon Michel Braunstein, si «les principales raisons avancées pour justifier le corps des professeurs de collège sont d'ordre pédagogique et sont fondées sur le fait que le collège doit avoir une identité propre dans ce domaine [...] cet argument est contestable sur divers points ${ }^{66}$. En aval du collège d'abord, la massification en cours du second cycle du second degré conduirait à ce que le problème de l'hétérogénéité des élèves touche également les lycées. En amont, "la logique qui sous-tend cette réforme et qui fait du collège une étape de transition entre l'école, où l'enseignement est assuré par un maître unique, et le lycée, où interviennent autant de professeurs qu'il y a de disciplines, sera bientôt battue en brèche par les autres réformes prévues dans l'enseignement primaire», par exemple l'introduction des langues vivantes ${ }^{67}$. Michel Braunstein en vient dès lors à prendre une position maximaliste consistant à défendre l'idée que la recomposition des champs disciplinaires ne peut seulement concerner les collèges, mais doit bel et bien s'appliquer à tous les niveaux d'enseignements.

Obtenue de haute lutte par $C$. Allègre contre l'avis de la majorité des membres du cabinet, la création du corps de professeurs de collège ne semble donc pas devoir survivre à l'opposition résolue du SNES et des défenseurs de l'enseignement secondaire classique, à laquelle font écho les objections formulées par plusieurs conseillers du ministre. De fait, à la suite de nouvelles rencontres officieuses avec les syndicats et de nouveaux arbitrages interministériels, L. Jospin va élaborer une nouvelle stratégie réformatrice. Constatant que «la FEN et le SNI sont revenus avec vigueur au discours idéologique de la parité entre professeurs et instituteurs » et que «le SNES s'arc-boute sur son refus du corps des collèges", son directeur de cabinet lui

65 Ibid.

66 Ibid.

67 Ibid. suggère en effet un "schéma [qui] permettrait d'apporter ce qui est le plus vigoureusement demandé (premier degré) et de retirer ce qui est le plus vigoureusement combattu (second degré) » ${ }^{68}$. Convaincu par cette analyse L. Jospin va donc acter une volte-face décisive en optant, d'un côté, pour la création d'un corps de professeurs des écoles bénéficiant d'un même niveau de recrutement et de rémunération que les certifiés et, de l'autre, pour l'abandon du corps de professeurs de collège. Validé lors d'une réunion interministérielle à laquelle assistent $M$. Rocard, Jean-Paul Huchon, Antoine Prost, Michel Charasse et B. Pêcheur ${ }^{69}$, ce marché est présenté à la direction du SNI-PEGC dès le 2 février lors d'une réunion confidentielle. Qualifiant la création du corps de professeurs des écoles de «changement historique ${ }^{70}$, L. Jospin explique donc qu'elle suppose en contrepartie le retrait du corps de professeurs de collège. Tout en invoquant «l'hostilité pratiquement générale dans le second degré » et en mettant en avant que le corps de collège est "considéré comme une déclaration de guerre par le SNES et le SNALC » ${ }^{71}$, il justifie sa décision en reprenant l'argumentation élaborée par ses conseillers. S'il affirme qu'il «croit toujours à la spécificité du collège » ${ }^{72}$, il explique en revanche qu'il «ne veut pas boucler statutairement avant d'avoir avancé sur la pédagogie $»^{73}$. Au terme d'un raisonnement intrinsèquement politique, il conclut en décrétant qu'il «ne peut avoir d'amis trop tièdes et d'ennemis trop irréductibles $»^{74}$, sous-entendant ainsi que le SNI-PEGC et la FEN sont moins attachés au corps de collège que le SNES et le SNALC n'y sont opposés. Mis face à un tel marché, J.-C. Barbarant va rester ferme sur ses positions en faisant valoir que «l'abandon du corps collège est un problème réel » qui revient à entériner le «maintien du grand second degré $»^{75}$. En effet, contrairement au ministre, il ne croit pas que la réforme statutaire et la réforme pédagogique puissent être dissociées. Mais tandis qu'il

68 «Réunion du 2 février», note d'Olivier Schrameck à L. Jospin,

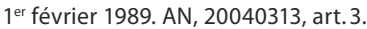

69 "Réunion chez Michel Rocard», notes manuscrites de L. Jospin, 2 février 1989. AN, 20040313, art. 3.

70 Notes manuscrites de L. Jospin prises lors de la réunion du 2 février. AN, 20040313, art.3.

71 lbid.

72 Notes manuscrites de Martine Le Gal prises lors de la réunion du 2 février. Archives du SNI-PEGC, boîte $n^{\circ} 681$.

73 Notes manuscrites de L. Jospin prises lors de la réunion du 2 février. AN, 20040313, art.3.

74 Notes manuscrites de Martine Le Gal prises lors de la réunion du 2 février. Archives du SNI-PEGC, boîte $n^{\circ} 681$.

75 lbid. 
explique à L. Jospin que «renoncer au corps collège, $c^{\prime}$ est renoncer à la spécificité $»^{76}$, ce dernier réaffirme quant à lui qu'«il faut maintenir l'objectif [...] mais sans se focaliser sur le corps de collège $»^{77}$. La réunion s'achève donc sur un constat de désaccord. Malgré l'organisation d'une journée de grève et de manifestation le 2 mars pour la «reconnaissance de la spécificité du collège et de l'enseignant qui y intervient ${ }^{78}$, le SNIPEGC ne parviendra pas à obtenir gain de cause.

En abandonnant la création du corps des professeurs de collège, le ministère de l'Éducation renonce donc à transformer l'organisation pédagogique de l'école moyenne en modifiant le statut des professeurs qui y exercent. Dans ces circonstances, le processus de réforme des contenus d'enseignement, initié à partir de l'été 1988 parallèlement aux discussions autour de la revalorisation, va redevenir le principal levier de cette transformation.

\section{Le rapport Bourdieu-Gros : recomposer les champs disciplinaires sans toucher au statut des enseignants?}

Malgré les craintes exprimées par L. Jospin lui-même quant au caractère politiquement explosif d'une telle entreprise, ses conseillers considèrent la rénovation des programmes ainsi que l'allègement et la refonte des grilles horaires imparties aux différentes disciplines comme un axe essentiel de la modernisation du système éducatif dans son ensemble (Clément, 2012). C'est le cas notamment du plus influent d'entre eux, $C$. Allègre, qui voit dans «le contenu et les modalités des enseignements [...] la source principale des dysfonctionnements de notre système éducatif $»^{79}$. Estimant que «les programmes scolaires (surtout au niveau du secondaire) sont encombrés et surchargés», il préconise de faire de "la stratégie d'allègement des horaires et des programmes ${ }^{80}$ le cœur de la politique pédagogique du ministère. Or, une telle stratégie ne semble pas pouvoir aboutir sans transformer profon-

76 Ibid.

77 Notes manuscrites de L. Jospin prises lors de la réunion du 2 février. AN, 20040313, art. 3.

78 L'École libératrice, $n^{\circ} 18,25$ février 1989.

79 «Faire du neuf avec du vieux. Un programme pour adapter notre éducation nationale aux temps futurs », note de $C$. Allègre à L. Jospin. AN, 20040313, art. 2. dément les cadres pédagogiques, c'est-à-dire les disciplines, qui, dans le second degré, organisent et structurent la transmission et l'appropriation des savoirs. Pour C. Allègre en effet, «notre système d'éducation est piloté par l'enseignement secondaire lui-même piloté par les classes préparatoires aux grandes écoles. La hiérarchisation des disciplines héritée d'Auguste Comte est confortée dans les grandes écoles, leurs hiérarchies, leurs programmes de préparation aux concours. Elle constitue la base sur laquelle s'appuie un corporatisme des disciplines toujours prêt à se mobiliser contre toutes réformes ${ }^{81}$.

\section{Réformer le curriculum pour changer l'école}

Pour tenter de battre en brèche le poids des disciplines dans l'enseignement secondaire, $C$. Allègre va donc mettre progressivement sur pied un important dispositif de rénovation des contenus d'enseignement. Considérant que "seuls ceux qui ont la plus grande connaissance des matières ont la capacité de dire ce qu'il faut dans les programmes ${ }^{82}$, il installe ainsi plusieurs commissions dites «verticales» ou «thématiques» dont il confie la présidence, non pas à des inspecteurs généraux, mais à des enseignants-chercheurs parvenus au sommet de la hiérarchie scientifique. «Initiateur principal» de cette réflexion sur les contenus (Legrand, 1994, p. 175), C. Allègre se voit laisser par L. Jospin toute latitude pour agir comme il l'entend sur ce dossier. Ainsi, pour nommer les présidents de commission, il s'appuie sur ses propres réseaux tissés à la fois dans le cadre de son activité scientifique et à la tête du groupe des experts du Parti socialiste, et puise dans le vivier de personnalités déjà mobilisées dans les commissions installées par Alain Savary (Heurdier \& Clément, 2016). Portant initialement sur les mathématiques, l'économie, la physique, les sciences de la terre et de l'univers, la chimie, la biologie, I'histoire et la géographie et, enfin, le français, ces commissions sont ainsi présidées respectivement par Didier Dacunha-Castelle (professeur de mathématiques à l'université Paris Sud), Edmond Malinvaud (titulaire de la chaire d'analyse économique du Collège de France), Pierre Bergé (chef de service au Commissariat à l'énergie atomique), René Blanchet (professeur de géologie à l'uni-

81 Ibid.

82 Notes manuscrites de L. Jospin prise lors d'un «Séminaire sur le scolaire», 24 juin 1988. AN, 20040313, art. 1. 
versité de Nice-Sophia Antipolis), François Mathey (professeur à l'École polytechnique), Hubert Condamines (professeur à Paris 7 et chef de service à l'Institut Pasteur), Philippe Joutard (professeur à I'université d'Aix-Marseille 1) et Bertrand Poirot-Delpech (écrivain, journaliste et académicien). Or, en reproduisant la spécialisation et la division du travail scientifique caractéristique de l'enseignement supérieur et de la recherche, un tel dispositif tend à entrer en contradiction avec l'objectif de recomposition des champs disciplinaires. Ainsi, alors que dans le second degré, la physique et la chimie sont réunies, elles sont ici séparées. Il en va d'ailleurs de même pour la biologie et les sciences de la terre, comme pour l'économie et les sciences sociales. Aussi, et pour éviter de «laisser s'affronter en champ clos les représentants des disciplines » ce qui conduirait « inévitablement à l'augmentation du volume des programmes», C. Allègre propose, dans un second temps, de compléter le dispositif en instaurant une commission dite «horizontale», chargée, pour chaque niveau d'enseignement, de réfléchir aux horaires et à l'équilibre entre les différentes disciplines, «deux problèmes » qui «sont sans doute [...] les plus difficiles à résoudre ${ }^{83}$.

C'est ainsi que le 2 novembre 1988, L. Jospin écrit à P. Bourdieu pour lui confier, en binôme avec F. Gros ${ }^{84}$, la responsabilité de cette commission. Le contenu de la lettre du ministre traduit à la fois les volontés réformatrices du ministre, mais aussi l'anticipation du caractère conflictuel de l'entreprise. D'un côté, il y affirme en effet sa volonté de recomposer les champs disciplinaires et d'alléger les programmes : «dans une période où les connaissances augmentent vite, où les frontières entre disciplines se déplacent, où naissent de nouvelles disciplines, il n'est plus possible de perpétuer la reproduction à l'identique des schémas traditionnels et l'accumulation des contenus des programmes sans réexaminer les fondements et les finalités mêmes des enseignements ${ }^{85}$. Mais, de l'autre, il reconnaît immédiatement les difficultés de la tâche «intrinsèques d'abord car il est malaisé de définir de nouveaux équilibres ou de nou-

83 «Faire du neuf avec du vieux. Un programme pour adapter notre éducation nationale aux temps futurs », note de $C$. Allègre à L. Jospin. AN, 20040313, art. 2.

84 Titulaire de la chaire de biochimie cellulaire au Collège de France depuis 1973 et directeur de l'Institut Pasteur de 1976 à 1981, F. Gros a notamment été conseiller des Premiers ministres Pierre Mauroy et Laurent Fabius.

85 Lettre de L. Jospin à P. Bourdieu, 2 novembre 1988. Archives personnelles de P. Bourdieu. veaux contenus [...], psychologiques et sociologiques ensuite car la force des habitudes incite chaque discipline à conserver le statu quo ou à répondre à l'accroissement des connaissances par une augmentation des programmes ${ }^{86}$.

Le premier acte de Pierre Bourdieu en tant que président de cette commission dite «horizontale» est d'organiser une table ronde avec les présidents des commissions thématiques pour non seulement préciser les enjeux de leur mission commune, mais aussi "réfléchir au redécoupage éventuel des disciplines des Sciences humaines ${ }^{87}$. Les notes manuscrites prises par $P$. Bourdieu en vue de la préparation de cette réunion, qui a finalement lieu le 26 novembre à la fondation Hugot $^{88}$, constituent un document précieux en ce qu'elles permettent de montrer qu'il partage alors certains des présupposés essentiels guidant l'entreprise réformatrice voulue par $C$. Allègre.

Premièrement, ces notes attestent que Pierre Bourdieu et Claude Allègre ont en commun l'idée que I'organisation disciplinaire du système d'enseignement dans le second degré est un obstacle central à toute entreprise de modernisation. La critique du « corporatisme des disciplines [qui] porte à perpétuer des savoirs périmés ou dépassés, et des divisions, qui peuvent persister dans l'organigramme scolaire, [...] alors qu'elles n'ont plus cours dans l'ordre de la recherche» était d'ailleurs déjà un point clé des Propositions pour l'enseignement de l'avenir ${ }^{89}$. Trois ans plus tard, P. Bourdieu considère toujours ce corporatisme comme l'un des principaux facteurs de conservation s'opposant à la modernisation du système d'enseignement. Après avoir rappelé en ouverture de la table ronde que «la révision périodique des savoirs enseignés» était l'un des principes des Propositions de l'enseignement de

86 Ibid.

87 Lettre de C. Allègre à P. Bourdieu, 18 novembre 1988. Archives personnelles de P. Bourdieu.

88 La création de cette fondation du Collège de France date de 1977 et a été rendue possible grâce à un don de Jean-Pierre Hugot et de sa sœur Hélène qui ont légué au Collège un immeuble de près de 3000 mètres carrés situé rue de I'Université ainsi qu'un portefeuille d'actions.

89 Collège de France, Propositions pour l'enseignement de l'avenir. Paris: Presses du Palais Royal, 1985, p. 31. À la suite d'une initiative de Pierre Bourdieu, le président de la République a demandé, en février 1984, à l'assemblée des professeurs du Collège de France de réfléchir aux principes fondamentaux de l'enseignement de I'avenir. À l'origine de cette commande, Pierre Bourdieu a également été le principal artisan de la rédaction et de l'élaboration du texte finalement remis à François Mitterrand le 27 mars 1985 (Clément, 2013, p. 195-238). 
l'avenir du Collège de France, il met en effet l'accent sur ce point qu'il qualifie lui-même de "très important» : I'«inertie du système d'enseignement» s'explique par la présence d'«intérêts corporatifs accrochés à des disciplines et des savoirs périmés» qui font que « le système [...] change par addition et jamais par suppression ${ }^{{ }^{90}}$. Signe de la virulence de sa critique des disciplines, il va jusqu'à comparer la résistance au changement de certaines disciplines au «poujadisme des petits commerçants menacés $)^{91}$.

Deuxièmement, à l'instar de C. Allègre qui, dans sa note programmatique citée ci-dessus, considérait que la légitimité à déterminer les contenus de l'enseignement scolaire procédait de la consécration dans le champ scientifique, P. Bourdieu estime lui aussi qu'il faut «partir de la science qui bouscule sans cesse ces divisions, crée des mélanges, se situe à des intersections (histoire économique - économie historique) pour bousculer ces "divisions fossiles héritées du passé » que sont les «divisions scolaires »". En tant que chercheurs parvenus au sommet de la hiérarchie scientifique dans leurs spécialités respectives ${ }^{93}$, P. Bourdieu et $C$. Allègre s'accordent ainsi sur un autre point essentiel du dispositif de rénovation des programmes qui conduit à reléguer au second plan les membres des corps d'inspection ainsi que les enseignants et leurs organisations représentatives, qu'il s'agisse des syndicats ou des associations de spécialistes. Un autre document issu des archives de P. Bourdieu permet de confirmer l'existence de ce point de convergence entre P. Bourdieu et C. Allègre. II s'agit d'une lettre envoyée par I'un des participants à la table ronde du 26 novembre, lequel signifie à P. Bourdieu qu'il «n'arrive pas à supporter le discours d'Allègre" parce qu'il le perçoit, entre autres, comme une forme de «mépris du supérieur pour ce qui est en dessous $»^{94}$. Or, en marge de cette remarque, Pierre Bourdieu formule un jugement lapidaire prenant le contrepied de son correspondant et allant dans le sens de $C$. Allègre : «non. et

90 Notes manuscrites de P. Bourdieu en vue de la réunion du 26 novembre 1988. Archives personnelles de P. Bourdieu.

91 Ibid.

92 Ibid.

93 II n'est peut-être pas inutile de rappeler ici qu'au moment où il devient conseiller de L. Jospin, C. Allègre est d'ores et déjà lauréat de nombreuses distinctions internationales, comme le prix Crafoord (1986) de l'Académie royale des sciences de Suède qui est en quelque sorte l'équivalent du prix Nobel pour les disciplines qui n'en sont pas pourvues. après tout justifié $»^{95}$. Troisièmement, les annotations en marge de ce document laissent également à penser que la convergence entre $P$. Bourdieu et $C$. Allègre ne se limitait peut-être pas aux deux aspects mentionnés précédemment. En effet, tandis que l'auteur de la lettre dénonce le risque que les membres des commissions puissent servir de caution à une réduction des horaires et à un allègement des programmes, deux objectifs essentiels de C. Allègre tenant non pas à des considérations scientifiques mais à la nécessité d'économies budgétaires, P. Bourdieu, sans toutefois être dupe, semble quant à lui prêt à jouer le jeu. Il écrit ainsi dans la marge : «pourquoi pas? si pas d'accord, quitter $\rightarrow$ conclusion : projet de stratégie globale : (1) degré de divergence, si totale : sortir (2) si pas totale : sommesnous capables d'infléchir? $»^{96}$.

Malgré leur caractère très parcellaire qui rend leur interprétation sujette à caution, les rares indices laissés par P. Bourdieu dans ses archives permettant de se faire une idée des dispositions dans lesquelles il aborde sa mission tendent à montrer une réelle convergence de vue avec $C$. Allègre sur les présupposés et les principes essentiels de la réforme des programmes.

Cela étant, les archives de P. Bourdieu montrent également qu'au-delà de cet accord sur les présupposés et les principes, les modalités concrètes de la réforme, c'est-à-dire au premier chef les éventuels redécoupages des disciplines scolaires et les possibles allègements de programme, ne sont jamais abordées de manière précise, restant de fait à l'état d'hypothèses de travail très peu formalisées. À l'instar des membres du cabinet de L. Jospin qui ne se formulent, au conditionnel, que des pistes de réflexion (cf. supra), P. Bourdieu en reste lui aussi au stade des suggestions s'agissant de cette recomposition des champs disciplinaires qu'il appelle de ses vœux. S'inscrivant dans la continuité du projet fondateur des sciences économiques sociales, dont il fut l'un des acteurs en tant que membre de la commission présidée par Charles Morazé (Beitone, Dollo, Hemdane et al., 2013), P. Bourdieu évoque ainsi, sans rentrer dans les détails, une possible réunion des sciences sociales dans l'enseignement supérieur mais reste silencieux sur l'enseignement de second degré. Cependant, et c'est là un point décisif, il prend clairement position quant au principe qui devrait gui-

95 Annotations manuscrites de P. Bourdieu en marge de la lettre de Jean-Claude Chevalier du 23 décembre 1988. Archives personnelles de P. Bourdieu.

96 Ibid. 
der un tel redécoupage des disciplines scolaires. En effet, estimant que, "plus on descend dans les niveaux d'instruction plus l'intégration doit être large ${ }^{97}$, il se trouve de fait sur la même ligne que le SNI-PEGC et la FEN qui défendent elles aussi l'idée d'une spécialisation disciplinaire croissante des maîtres à mesure que I'on avance dans les degrés d'enseignement.

Ainsi, à l'instar des réformateurs sociaux du XIX ${ }^{e}$ siècle, étudiés par Christian Topalov, ce que partagent $C$. Allègre, $P$. Bourdieu et la majorité UID de la FEN, c'est bien une partie d'un certain sens commun réformateur, c'est-à-dire "un langage partagé, une mise en forme des problèmes et un horizon des possibles sur lesquels s'établit l'accord», mais qui «n'impliquent nullement un consensus sur les modalités effectives de l'action, encore moins sur ces fins dernières» (Topalov, 1999, p.44).

Malgré tout, la discussion qui s'engage sur ces bases lors de la table ronde du 26 novembre aboutit finalement à modifier significativement le dispositif de départ dans le sens d'une plus grande intégration disciplinaire. Renouant avec le projet ancien d'ouverture de l'histoire aux sciences sociales (Legris, 2010), la commission «histoire-géographie» se voit adjoindre les «sciences sociales». Celle consacrée au «français » a été renommée «langues et littératures » et se trouve désormais présidée par Bernard Lortholary et Jean-Claude Chevalier, ancien président de la commission de réflexion sur le français mise en place sous le ministère Savary et ancien collègue de Pierre Bourdieu à l'université de Lille dans les années $1960^{98}$. Enfin est créée une nouvelle commission intitulée «philosophie-épistémologie » dont la présidence revient à Jacques Bouveresse et Jacques Derrida, deux proches de Pierre Bourdieu, hostiles à l'enseignement traditionnel de la philosophie tel qu'il se pratique alors dans les classes de terminale.

\section{La critique des disciplines : clé de voûte du rapport Bourdieu-Gros}

Alors même que, au mois de février 1989, les premières fuites émanant des commissions et la publication par I'hebdomadaire Informations ouvrières des «nouvelles grilles d'emploi du temps scolaire, mises au point par le cabinet du ministre Jospin et proposées aux diverses

97 Ibid.

98 À voix nue, France Culture, 12 mars 2007. commissions installées par le ministre ${ }^{99}$ suscitent des réactions hostiles au sein de certaines disciplines inquiètes pour leur avenir et un embarras croissant chez P. Bourdieu ${ }^{100}$, celui-ci n'entend cependant pas renoncer à énoncer publiquement les prises de position qui lui tiennent à cœur. C'est ainsi que la critique de l'organisation disciplinaire de l'enseignement constitue sans aucun doute la colonne vertébrale des Principes pour une réflexion sur les contenus de l'enseignement qu'il remet à L. Jospin et qui sont rendus publics au début du mois de mars 1989. D'ailleurs, pour bien marquer le caractère contingent de l'état présent des divisions qui structurent la transmission de la culture scolaire, le mot discipline est toujours employé avec des guillemets.

Présente dès le préambule qui proclame que «ces propositions [...] porteront essentiellement restructuration des divisions du savoir $»^{101}$, cette mise en question des disciplines traverse le texte de part en part. Le deuxième principe développe ainsi l'idée selon laquelle la finalité dernière de l'enseignement ne devrait pas être l'acquisition de connaissances factuelles ou de savoirs, mais bien, d'une part, de "savoirfaire fondamentaux» - assimilés ici aux techniques et aux méthodes du travail intellectuel dans un style qui fait très nettement écho au plaidoyer pour la pédagogie rationnelle qui conclut Les Héritiers - et, d'autre part, de «modes de pensées fondamentaux»- «déductif», "expérimental», "historique», «réflexif et critique $»^{102}$. Cette primauté accordée à ces savoir-faire et ces modes de pensées fondamentaux conduit dès lors à reconsidérer la fonction et l'utilité des différentes disciplines en se demandant comment chacune d'elles peut «contribuer à la transmission des différents modes de pensée, et si certaines spécialités ne sont

99 Informations ouvrières, nº 1404, 8-15 février 1989. Informations ouvrières est l'organe du Mouvement pour un parti des travailleurs, une organisation trotskyste lambertiste de laquelle proviennent certains des militants à l'origine de la création, dans la première moitié des années 1980, des syndicats enseignants rattachés à Force ouvrière.

100 À Bernard Lortholary, qui vient de démissionner de la présidence de la commission "langues et littératures» à cause de son manque d'indépendance, Pierre Bourdieu répond : «je ne comprends que trop... Pardonne-moi de t'avoir embarqué dans cette galère». Il envoie par la suite un mot à Joseph Goy, conseiller de L. Jospin, indiquant : "ça m'inquiète un peu. Peux-tu m'appeler le plus vite possible? ». Réponse de P. Bourdieu à B. Lortholary et mot de P. Bourdieu à J. Goy. Archives personnelles de P. Bourdieu. 101 lbid., p. 3.

102 Principes pour une réflexion sur les contenus de l'enseignement, s.l., s.n., 1989 , p. 6. 
pas mieux placées, par toute leur logique et leur tradition, pour assurer l'apprentissage réussi de l'un ou l'autre d'entre eux $»^{103}$. Si I'on accepte ce principe, qui préfigure l'idée selon laquelle il peut y avoir en pédagogie comme en droit une hiérarchie des normes, alors les disciplines d'enseignement ont vocation à être subordonnées à des objectifs de formation plus généraux et à n'exister que si et seulement si elles sont en mesure de faire la preuve qu'elles sont les mieux à même de contribuer à la réalisation de ces objectifs. Ce principe de base étant posé, la critique des disciplines va s'exprimer plus particulièrement dans les cinquième et sixième principes. La diversification des formes d'enseignement, qui verrait élèves et enseignants interagir dans plusieurs types de configurations pédagogiques, est ainsi justifiée au motif qu'elle permettrait de remettre en cause «l'enseignement actuel, encyclopédique, additif et cloisonné » et d'«échapper à la simple comptabilité par "discipline" qui est un des obstacles majeurs à toute transformation réelle des contenus des enseignements $»^{104}$. De la même manière, l'appel à ce que les professeurs coordonnent leurs actions, par exemple en donnant des heures de cours en commun, est présenté comme une contribution à "l'effort, absolument nécessaire, pour repenser et surmonter les frontières entre les "disciplines" $»^{105}$. Le texte affirme enfin que «le souci de renforcer la cohérence des enseignements devrait conduire à favoriser les enseignements donnés en commun par des professeurs de différentes spécialités et même à repenser les divisions en "disciplines", en soumettant à l'examen certains regroupements hérités de l'histoire et en opérant, toujours de manière progressive, certains rapprochements imposés par l'évolution de la science $»^{106}$.

Salué comme un «excellent travail » ${ }^{107}$ dans le bulletin de la FEN, approuvé sans réserve par le SNI$\mathrm{PEGC}^{108}$ mais accueilli de manière très critique par le SNES ${ }^{109}$, le rapport dit «Bourdieu-Gros» constitue une contribution décisive dans la manière de problématiser la réforme des programmes. II apporte en effet une caution scientifique, d'un côté, à l'idée que l'organisation disciplinaire de l'enseignement doit être revue de

103 lbid.

104 Ibid., p. 10.

105 Ibid., p. 12

106 lbid., p. 14

107 L'Enseignement public, n57, mai 1989.

108 Note de Jeanne Finet, secrétaire nationale du SNI-PEGC chargée fond en comble et, de l'autre, à l'idée que les savoirs disciplinaires doivent être subordonnés à des objectifs généraux de formation.

\section{Le rapport Bourdieu-Gros : un essai non transformé}

Malgré l'intervention initiale de P. Bourdieu visant à ce que l'intitulé et le périmètre des commissions thématiques aillent dans le sens d'une plus grande intégration disciplinaire, leur réflexion tend à nouveau à s'inscrire dans les divisions héritées du passé. Or ceci entre en contradiction directe avec le contenu des Principes. C'est précisément le sens de la lettre qu'adresse P. Bourdieu aux présidents des différentes commissions ainsi qu'à C. Allègre à la fin du mois de mai 1989. Après avoir rappelé le mandat qui leur a été donné, il écrit ainsi : «il me paraît donc tout à fait regrettable que nous soyons retournés en fait, pour la rédaction des rapports, à la division la plus traditionnelle en disciplines : philosophie, mathématiques, français, langues, physique, chimie, géologie, histoire, géographie, économie - il ne manque, cela ne vous aura pas échappé, que la sociologie... Convaincu qu'un des principes majeurs du renouvellement des contenus et des méthodes d'enseignement consiste dans la mise en question des disciplines (de leur existence en certains cas, de leurs frontières en d'autres cas), je ne puis, pour ma part, que me désolidariser par avance d'un rapport final qui accepterait en fait ces divisions : comment, en effet, combattre le corporatisme professoral qui est, on le sait bien, un des obstacles majeurs au renouveau pédagogique, si une commission qui prétend ne reconnaître d'autres principes que ceux de la science cède par avance sur un point de cette importance? $»^{110}$ Mais si cette lettre est l'occasion pour P. Bourdieu de réaffirmer la validité de la critique de l'organisation disciplinaire de l'enseignement et de ses effets, elle a comme objectif premier de réclamer qu'une traduction pratique soit donnée aux Principes. Afin d'éviter que les rapports des commissions ne reproduisent et n'entérinent les divisions disciplinaires, $P$. Bourdieu plaide pour leur regroupement en «cinq grandes unités (langues et littératures, philosophie, sciences sociales,

110 Lettre de P. Bourdieu à F. Gros, C. Allègre, P. Bergé, R. Blanchet, J. Bouveresse, J.-C. Chevalier, H. Condamines, D. Dacunha-Castelle, J. Derrida, P. Joutard, E. Malinvaud et F. Mathey, 29 mai 1989. Archives personnelles de P. Bourdieu. 
mathématiques et sciences expérimentales) $»^{111}$. Surtout, il préconise que soit clairement énoncé de quelle manière rendre les Principes effectifs, c'est-à-dire en "donnant des exemples de choses à supprimer, qu'il s'agisse de disciplines entières ou de secteurs de telle ou telle des disciplines traditionnelles», en prenant position sur les modalités pratiques des examens ou encore en explicitant quels sont les «modes de pensée fondamentaux» et, "corrélativement, quels sont les enseignements secondaires, superflus ou inutiles qui devraient être réduits ou supprimés $»^{112}$.

Malheureusement, les archives auxquelles j'ai pu avoir accès ne permettent pas de reconstituer de manière précise les rapports de force qui ont pu exister non seulement au sein des différentes commissions, mais aussi entre elles et entre leurs présidents. Malgré tout, les réponses à l'appel de P. Bourdieu laissent penser que sa position n'était pas majoritaire, ce qui I'a conduit à se désengager du dispositif de réforme des contenus. Dans une lettre du 4 juin, Pierre Bergé, président de la commission de physique, lui écrit pour lui faire de ses doutes quant à la possibilité de réaliser une synthèse permettant de dépasser la simple juxtaposition de contributions disciplinaires : «En réponse à votre lettre du 31 mai je vous fais savoir que, comme vous, je pense qu'il serait important, pour marquer notre action achevée, de rendre un rapport de synthèse fin juin. Je vois néanmoins deux difficultés de nature très différente à cette entreprise. L'une est que vous souhaitez regrouper la substance de ces rapports disciplinaires ("verticaux") en cinq grandes unités : je crains fort une réticence de certain(s) président(s) à placer leur discipline dans ces unités. Ceci peut handicaper la réalisation de notre synthèse. Beaucoup plus préoccupant : quel rédacteur habile et puissant va se charger de faire - en un très court laps de temps - la difficile synthèse des travaux des commissions, si vousmême vous mettez en retrait comme j'ai cru le comprendre lors de notre dernière réunion? »113 De la même manière, dans un petit mot manuscrit daté du 9 juin 1989, Pierre Baqué, futur membre du CNP que L. Jospin a chargé de réfléchir à l'enseignement des arts plastiques parallèlement aux travaux des commissions thématiques, dresse lui aussi un constat d'échec quant à la possibilité de voir le travail des commissions

111 Ibid.

112 lbid.

113 Lettre de P. Bergé à P. Bourdieu, 4 juin 1989. Archives personnelles de P. Bourdieu. déboucher sur des prises de position résolument novatrices : «J'approuve entièrement ta lettre du 29 mai 89. Les commissions actuelles fonctionnent de façon traditionnelle reproduisant les démarches dont souffre tant le système éducatif. J'étais le seul à ne pas protester contre l'absence de commissions spécifiques aux enseignements artistiques. Cet oubli d'Allègre me semblait positif sous réserve que les autres ne tombent pas dans les erreurs passées. Ce qui est fait $»^{114}$.

Mais le signe le plus tangible de l'échec de P. Bourdieu à imposer son point de vue est que le rapport de synthèse qu'il a pu appeler de ses vœux ne voit pas le jour. Ce sont finalement plusieurs rapports disciplinaires qui seront remis au cabinet du ministre en juin et en juillet 1989 et qui seront désormais censés alimenter les travaux du futur CNP et servir de base aux colloques académiques organisés à l'automne 1989.

\section{Conclusion}

La courte période allant de la réélection de F. Mitterrand à la fin de l'année 1989 apparaît donc décisive pour l'histoire du système d'enseignement en général et pour des réformes du curriculum en particulier.

Sur le plan syndical d'abord, le statu quo statutaire dans le second degré résultant de l'abandon du corps des professeurs de collège signifie la faillite de la stratégie élaborée par la tendance UID en accord avec le secrétariat national à l'éducation du PS. Alors que la création de ce nouveau corps devait être l'instrument lui permettant de conserver le contrôle de la FEN face à la progression de la minorité UA, son abandon va ouvrir la voie à un nouvel ordre des choses syndical avec la scission de la "forteresse enseignante» puis l'émergence de la FSU (Fédération syndicale unitaire) qui deviendra bientôt la première organisation syndicale dans l'Éducation nationale.

Mais cette décision aboutit également à trancher une question statutaire essentielle laissée en suspens par les réformes des structures pédagogiques des années 1950-1970. Si celles-ci ont finalement abouti au terme d'un long processus, à ce que, aujourd'hui, la quasi-totalité des élèves des collèges soient scolarisés au sein d'une filière unique ${ }^{115}$, elles n'avaient pas mis

114 Lettre de P. Baqué à P. Bourdieu, 9 juin 1989. Archives personnelles de P. Bourdieu.

115 Lointaines héritières des «filières scolaires de l'échec» (Chevallier, 1986), les classes de troisième préparatoires aux formations professionnelles (dites « $3^{\mathrm{e}}$ prépa-pro»), les classes de troisième des sections d'enseignement général et professionnel adapté (SEGPA) 
fin à la concurrence entre enseignants issus du primaire et du secondaire pour le monopole de l'enseignement dans les collèges. Or, en entérinant l'état de fait résultant de l'arrêt du recrutement des PEGC, I'abandon du corps des professeurs de collège débouche sur l'instauration d'un nouvel ordre des choses statutaires qui tient toujours aujourd'hui.

Précisément parce qu'elle débouche sur ce nouvel ordre des choses, cette période apparaît enfin décisive pour l'histoire des contenus d'enseignement. Dès lors qu'ont été fixés les structures pédagogiques et le statut des maîtres des collèges, c'est à nouveau sur le terrain pédagogique que vont se (re)jouer les luttes scolaires ayant pour enjeu le positionnement de cette école moyenne de masse dans le système d'enseignement. Dans cette configuration, repenser la place des disciplines en tant que principe organisateur de la transmission des savoirs s'impose ainsi comme l'un des

et les dispositifs d'initiation aux métiers en alternance (DIMA) accueillent respectivement en 2013-2014 34 160, 25313 et 5769 élèves ce qui représente au total $8 \%$ des élèves de troisième (sources : Inspection générale de l'Éducation nationale (2015), Bilan et perspectives relatifs à la classe de troisième préparatoire aux formations professionnelles. Rapport n²015-069 et Ministère de l'Éducation nationale de l'Enseignement supérieur et de la Recherche [2014 et 2015], Repères et références statistiques).

\section{Bibliographie}

AEBISCHER S. (2010). "Mettre l'élève et le management au centre du système». Sociologie d'un moment réformateur - le ministère Jospin (1988-1989). Thèse de doctorat, science politique, université Lyon 2.

AUBERT V., BERGOUNIOUX A., MARTIN J.-P. \& MOURIAUX R. (1985). La forteresse enseignante : la Fédération de l'Éducation nationale. Paris : Fayard/Fondation Saint-Simon.

BALLAND L. (2009). Une sociologie politique de la crise de l'École. De la réussite d'un mythe aux pratiques enseignantes. Thèse de doctorat, science politique, université Paris 10-Nanterre.

BEITONE A., DOLLO C., HEMDANE E. \& LAMBERT J. (2013). «1. Les sciences économiques et sociales : Origine et dynamique d'une discipline scolaire». In A. Beitone, C. Dollo, E. Hemdane \& J. Lambert, Les sciences économiques et sociales : Enseignement et apprentissages. Louvain-laNeuve : De Boeck, p. 9-42.

BECHER T. (1989). Academic Tribes and Territories. Intellectual Enquiry and the Culture of Discipline. Milton Keynes : The Society for Research into Higher Education / Open University Press.

BEZES P. (2009). Réinventer l'État. Les réformes de l'administration française (1962-2008). Paris : PUF. principaux leviers de la transformation du collège. C'est ce dont témoignent notamment les travaux du CNP, la mise en place du socle commun de connaissances et de compétences ou encore l'introduction des Enseignements pratiques interdisciplinaires à la rentrée 2016. Si l'action du Conseil national des programmes commence à être bien documentée - grâce à un certain nombre de publications (Legris, 2010; Clément, 2012; Heurdier \& Clément, 2016) ${ }^{116}$ et aux recherches menées dans le cadre du séminaire d'Antoine Prost - et si la genèse du socle commun a d'ores et déjà donné lieu à certains travaux (Clément, 2013; Lelièvre, 2016), il resterait donc à montrer en quoi les tentatives récentes de l'organisation pédagogique du collège participent, ou non, de ce mouvement ancien consistant à faire de la rénovation des programmes le principal levier de la réforme de l'école.

Pierre Clément

Université de Rouen, Centre interdisciplinaire de recherche normand en éducation et formation (CIRNEF) pierclement@gmail.com

116 La revue Carrefours de l'éducation doit publier en 2019 un dossier consacré au CNP.

BOURDIEU P. (1976). "Le champ scientifique». Actes de la recherche en sciences sociales, $\mathrm{n}^{\circ} 2-3, \mathrm{p} .88-104$.

BOURDIEU P. (1991). "Le champ littéraire». Actes de la recherche en sciences sociales, $n^{\circ} 89$, p.3-46.

BOURDIEU P. (1997). Les usages sociaux de la science. Pour une sociologie clinique du champ scientifique. Paris : INRA Éditions.

BOURDIEU P. \& PASSERON J.-C. (1970). La reproduction. Éléments pour une théorie du système d'enseignement. Paris : Éd. de Minuit.

BOUTIER J., PASSERON J.-C. \& REVEL J. (dir.) (2006). Qu'est-ce qu'une discipline? Paris : Éd. de l'EHESS.

BRUCY G. (2003). Histoire de la FEN. Paris : Belin.

CHAPOULIE J.-M. (2010). L'école d'État conquiert la France. Rennes : Presses universitaires de Rennes.

CHEVALLIER P. (1986). "Les filières scolaires de l'échec ». Revue française de pédagogie, $\mathrm{n}^{\circ} 77, \mathrm{p} .39-46$.

CLÉMENT P. (2012). «Le Conseil national des programmes (1985-1994) : I'institutionnalisation chaotique d'une entreprise réformatrice». Politix, n ${ }^{\circ}$ 8, p. 85-107.

CLÉMENT P. (2013). Réformer les programmes pour changer l'école? Une sociologie historique du champ du pouvoir sco- 
laire. Thèse de doctorat, sociologie, université de Picardie.

D'ENFERT R. \& KAHN P. (dir.) (2011). Le temps des réformes. Disciplines scolaires et politiques éducatives sous la Cinquième République : les années 1960. Grenoble : Presses universitaires de Grenoble.

FORQUIN J.-C. (2008). Sociologie du curriculum. Rennes : Presses universitaires de Rennes.

FRAJERMAN L. (2003). L'interaction entre la Fédération de l'Éducation nationale et sa principale minorité, le courant "unitaire», 1944-1959. Thèse de doctorat, histoire, université Paris 1.

GEAY B. (1994). La fin de l'univers primaire, les instituteurs français et la représentation syndicale. Thèse de doctorat, sociologie, EHESS.

GEAY B. (2005). Le syndicalisme enseignant. Paris : La Découverte.

GUSFIELD J. R. (2009). La culture des problèmes publics. L'alcool au volant : la production d'un ordre symbolique. Paris : Economica.

HEURDIER L. \& CLÉMENT P. (2016). «Aux origines du Conseil national des programmes (1983-1990) : instruments et acteurs d'une nouvelle gouvernance des contenus d'enseignements ». Revue française de pédagogie, n`195, p. 5-24.

LEGRAND A. (1994). Le système E. L'École... de réformes en projets. Paris : Denoël.

LEGRIS P. (2010). L'écriture des programmes d'histoire en France (1944-2010). Sociologie historique d'un instrument d'une politique éducative. Thèse de doctorat, science politique, université Paris 1.

LELIÈVRE C. (2011). «Quid de l'école du peuple et des différentes strates du système éducatif?». Carrefours de l'éducation, hors série, $\mathrm{n}^{\circ} 2,85-95$.

LELIEVRE C. (2016). «Le“socle commun"». Carrefours de l'éducation, $n^{\circ} 41$, p. 169-182.
MUSGROVE F. (1968). «The Contribution of Sociology to the Study of Curriculum ». In J. F. KERR (dir.), Changing the Curriculum. Londres : University of London Press, p. 96-109.

POULLAOUEC T. \& LEMÊTRE C. (2009). «Retours sur la seconde explosion scolaire ». Revue française de pédagogie, $\mathrm{n}^{\circ} 167$, p. 5-11.

PROST A. (1968). L'enseignement en France 1800-1967. Paris : Armand Colin.

PROST A. (2004). Histoire générale de l'enseignement et de l'éducation en France. Tome IV. L'école et la famille dans une société en mutation (depuis 1930). Paris : Perrin.

PROST A. (2007). «Une histoire des collèges. III. La réforme : quels murs et quels maîtres?» In Regards historiques sur l'éducation en France $X I X^{e}-X X^{e}$ siècles. Paris : Belin, p. 145-148.

ROBERT A. (2010a). "La“nébuleuse" de la réforme Haby (19731980) ». In L'école en France de 1945 à nos jours. Grenoble : Presses universitaires de Grenoble, p. 95-120.

ROBERT A. (2010b). «Le SNI et le corps des PEGC: du trouble à la conversion; une recomposition identitaire (19541976) ». In L. Frajerman, F. Bosman, J.-F. Chanet \& J. Girault (dir.), La Fédération de l'Éducation nationale (1928-1992). Histoire et archive en débat. Villeneuve-d'Ascq : Presses universitaires du Septentrion, 2010, p.123-134.

ROBERT A. (2016). «Le"projet de modernisation du système éducatif" : prémices proches et lointaines, contexte, processus d'élaboration (été 1974-février 1975) ». In L. Gutierrez \& P. Legris, Le collège unique. Éclairages socio-historique sur la loi du 11 juillet 1975. Rennes : Presses universitaires de Rennes, p.51-69.

TOPALOV C. (1999). Laboratoires du nouveau siècle. La nébuleuse réformatrice et ses réseaux en France (1880-1914). Paris : Éditions de l'École des hautes études en sciences sociales. 
